\title{
Um método para previsão de sobrecarga transiente em sistemas computacionais por meio de modelos dinâmicos obtidos empiricamente
}

Helder Jefferson Ferreira da Luz 



\title{
Um método para previsão de sobrecarga transiente em sistemas computacionais por meio de modelos dinâmicos obtidos empiricamente
}

\author{
Helder Jefferson Ferreira da Luz
}

Orientador: Prof. Dr. Francisco José Monaco

\begin{abstract}
Dissertação apresentada ao Instituto de Ciências Matemáticas e de Computação - ICMC-USP, como parte dos requisitos para obtenção do título de Mestre em Ciências - Ciências de Computação e Matemática Computacional. VERSÃO REVISADA
\end{abstract}


Ficha catalográfica elaborada pela Biblioteca Prof. Achille Bassi e Seção Técnica de Informática, ICMC/USP, com os dados fornecidos pelo(a) autor(a)

Luz, Helder Jefferson Ferreira da

L979m Um método para previsão de sobrecarga transiente em sistemas computacionais por meio de modelos

dinâmicos obtidos empiricamente / Helder Jefferson Ferreira da Luz; orientador Francisco José Monaco. - São Carlos, 2014.

$41 \mathrm{p}$.

Dissertação (Mestrado - Programa de Pós-Graduação em Ciências de Computação e Matemática

Computacional) -- Instituto de Ciências Matemáticas e de Computação, Universidade de São Paulo, 2014.

1. Identificação de sistemas. 2. Análise transiente. 3. Planejamento de capacidade. 4. Sistemas adaptativos. 5. Avaliação de desempenho. I. Monaco, Francisco José, orient. II. Título. 
O sucesso é ir de fracasso em fracasso sem perder o entusiasmo.

Winston Churchill 

Dedico este trabalho aos meus pais, Humberto e Cláudia, que me apoiaram durante todo o meu percurso. 



\section{Agradecimentos}

Primeiramente aos meus pais, por me ajudarem a trilhar esse caminho. Aos meus professores de graduação, em especial, prof. Dacêncio e prof. Botega, que não apenas me orientaram nos quatro anos de graduação, mas também me incentivaram a cursar o mestrado. Aos meus amigos desde a graduação, Davi Nakano, Fábio Jorge, Fernando Sato, Guilherme Cruz, Raphael Honda e Richard Frank, por todas as conversas e distrações que certamente me ajudaram a chegar aqui.

Ao Edwin, Lourenço, Pedro, Renê, Bruno e a todos que me auxiliaram durante o mestrado, que muitas vezes tiveram que abrir mão do tempo com as suas famílias para tirar minhas dúvidas e me ajudar a resolver os diversos problemas enfrentados no caminho. E claro, ao meu orientador e professor Francisco José Monaco que me ajudou e sempre enfrentou os prazos tão limitados que eu lhe apresentava. 

Ste trabalho apresenta um método empírico para previsão de sobrecargas transientes em sistemas computacionais por meio de modelagem dinâmica. A técnica, baseada em aproximações lineares e invariantes no tempo, tem como objetivo identificar a capacidade de um sistema computacional absorver variações na carga de trabalho. Experimentalmente, a identificação dessa capacidade do sistema pode ser feita por meio de técnicas de avaliação de desempenho, em que a abordagem prevalente é a estimação da capacidade estática em regime estacionário de operação, observando-se o desempenho sob demanda constante. Entretanto, essa avaliação não considera o regime transiente do sistema, i.e durante o período de restabelecimento ao regime estacionário após uma perturbação, e durante o qual, o esforço exigido pode ser bastante diverso, e potencialmente acima daquele apurado sob condições de regime estacionário. A proposta deste trabalho é a formulação de uma metodologia para avaliação de desempenho em regime transiente em sistemas computacionais sob carga de trabalho variável e que forneça informação para o dimensionamento de recursos e políticas de controle de admissão que evitem sobrecargas por efeitos transitórios. A metodologia baseia-se na parametrização de um modelo dinâmico a partir de ensaios experimentais, considerando perturbações bruscas e de longa duração, e os resultados são avaliados por comparação das predições do modelo em relação aos objetivos por simulação ou aferição. 

7 His research work introduces an empirical method for the prediction of transient overloads in computer systems by means of dynamical modeling. The technique, based on linear time-invariant approximations, aims at identifying the computer system's capacity in absorbing variations on the workload. Experimentally, this capacity identification can be carried out from performance evaluation methods, whose prevalent approach is the estimation of the static capacity under stationary operational regime, by observing the performance under constant demand. Nevertheless, this kind of evaluation does not take into account the system's transient regime, i.e. the period during of the restablishment to the stationary regime after the perturbation, and within which, the effort required from the systems may be diverse and potentially superior to that measured under the stationary condition. This work proposes the formulation of a methodology for performance evaluation in transient regime of computer systems submitted to variable workloads, aimed at providing information for dimensioning or resources and design of admission control policies capable of avoiding overloads due to transitory effects. The methodology relies on the parametrization of a dynamical model obtained from experimental procedures, considering abrupt, long-lasting distrubances, and the results are evaluated through comparison of the model prediction with the simulated system. 

Resumo

$\begin{array}{lll}\text { Abstract } & \text { iii }\end{array}$

Lista de Siglas $\quad$ xi

1 Introdução $\quad \mathbf{1}$

1.1 Avaliação de capacidade estática e capacidade dinâmica . . . . . . . . . . . . . 2

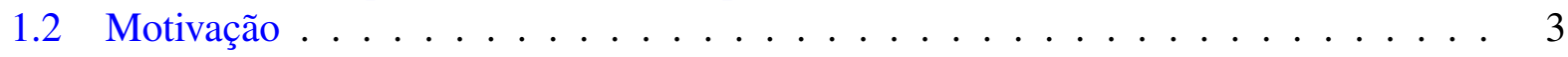

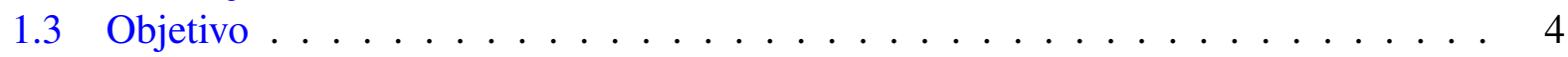

2 Revisão $\quad 5$

2.1 Resultados anteriores no grupo de pesquisa . . . . . . . . . . . . . . . 6

2.2 Arquitetura de Nobile . . . . . . . . . . . . . . . . 7

3 Metodologia 9

3.1 Resultados Preliminares do Estudo . . . . . . . . . . . . . . . . . . . . . . . . . . . . .

3.2 Estudo de caso: arquitetura de Nobile . . . . . . . . . . . . . . . 12

4 Desenvolvimento 17

4.1 Abordagem e hipóteses . . . . . . . . . . . . . . . . . . . . . . . . . . . . . . . .

4.2 Procedimento experimental . . . . . . . . . . . . . . . . . 19

4.2.1 Experimentos para coleta de dados . . . . . . . . . . . . . 20

4.3 Resposta ao degrau . . . . . . . . . . . . . . . . . . . . . . . . . . . . . . . . . . . .

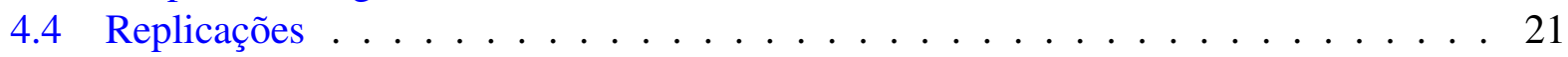

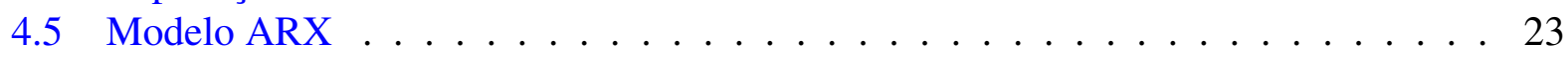

4.6 Função de transferência . . . . . . . . . . . . . . . . . . . . . . . . 24

4.7 Avaliação do modelo . . . . . . . . . . . . . . . . . . . . 26

4.8 Tratamento de sinais . . . . . . . . . . . . . . . . . . . . 26

4.9 Avaliação do modelo com filtro . . . . . . . . . . . . . . . . . . 27

4.10 Caracterização de sobrepassagem e tempo de assentamento . . . . . . . . . . . . 29

5 Conclusões $\quad 33$ 



\section{Lista de Figuras}

2.1 Diagrama simplificado da arquitetura de controle de Nobile (Nobile, 2013). . . . 7

3.1 Etapas para modelagem de caixa preta de um sistema dinâmico. . . . . . . . . . . 11

3.2 Intervalo e variação de entradas aplicados à modelagem do sistema . . . . . . . . . . 13

3.3 Saída observada do sistema em resposta à variação da entrada . . . . . . . . . . . . . . 13

3.4 Comparação da resposta real medida com a resposta prevista pelo modelo . . . . . 15

4.1 Diagrama do sistema. . . . . . . . . . . . . . . . . . . 17

4.2 Carga de trabalho $(W)$ de uma única replicação . . . . . . . . . . . . . . . . . 22

4.3 Número de máquinas virtuais $(N)$ de uma única replicação . . . . . . . . . . . . . 22

4.4 Carga de trabalho $(\mathrm{W})$ imposta ao sistema. . . . . . . . . . . . . . . 23

4.5 Número de máquinas virtuais previstas pelo controlador. . . . . . . . . . . . . . 24

4.6 Saída prevista pela função de transferência e saída real do sistema . . . . . . . . . 26

4.7 Ruído em W após utilizar o filtro em relação a W sem filtro. . . . . . . . . . . . . 27

4.8 Saída prevista pela função de transferência e saída real. . . . . . . . . . . . . . . . 28

4.9 Saída prevista pela função de transferência contraposta à saída real. . . . . . . . . . 28

4.10 Exemplo de resposta de um sistema de $2^{\mathrm{a}}$ ordem a uma entrada degrau. . . . . . . . 30 



\section{Lista de Tabelas}

3.1 Valores de $R^{2}, R M S E$ e correlação entre entrada e erro residual para o modelo estimado. . . . . . . . . . . . . . . . . . . 16

4.1 Valores de $R^{2}, R M S E$ e correlação entre entrada e a saída do modelo estimado. . . 29

4.2 Comparação de características do modelo analítico com o mensurado do sistema. . 31 

ARX - AutoRegressive with eXternal input

GSDPC - Grupo de Sistemas Distribuídos e Programação Concorrente

FIR - Finite Impulse Response

FT - Função de Transferência

LTI - Linear Time Invariant

QoS - Quality of Service

RMSE - Root Mean Square Error

RTU - Real Time Unit

STU - Sample Time Unit

SLA - Service Level Agreement 
Um dos componentes importantes em uma arquitetura de provisão de qualidade de serviço (QoS) em sistemas computacionais é a política de controle de admissão, responsável por evitar que uma carga de trabalho excessiva degrade o desempenho do sistema a um nível insuficiente para cumprir os acordos de nível de serviço (SLA). Em função da carga imposta pelo fluxo de requisições recebido e na capacidade instantânea do sistema em absorver a demanda sem comprometer o serviço oferecido a outros usuários, a política de admissão deve decidir sobre a aceitação ou descarte da requisição, a ou renegociação do acordo de serviço.

A atuação do controle de admissão tem o objetivo de evitar a sobrecarga do sistema por uma demanda que exceda sua capacidade. Logo, seu projeto se baseia no conhecimento dessa capacidade para dimensionamento de recursos e ajuste de parâmetros do algoritmo de tomada de decisão. A capacidade de um sistema computacional pode ser estimada mediante o emprego de técnicas de avaliação de desempenho, as quais podem ser baseadas em aferição ou modelagem (Jain, 1991).

A primeira forma, a aferição, consiste na realização de medidas sobre o sistema real. Tem a vantagem da observação empírica do real comportamento, incluindo o efeito de todos os fatores que influenciam no resultado. Como desvantagem, ressalva-se a potencial dificuldade de instrumentação, necessidade de replicações para validação estatística e de considerações sobre a influência da medida no próprio resultado. Na modelagem, por sua vez, utiliza-se uma abstração do sistema que congregue as características que se acredita poderem influenciar o resultado. $\mathrm{O}$ modelo pode ser abordado tanto analiticamente quanto por meio de simulação. Um modelo analítico corresponde a uma representação matemática das propriedades do sistema do qual podem ser derivadas conclusões teóricas. Um modelo de simulação corresponde a uma implementação equivalente do sistema por meio de um protótipo de propriedades supostamente similares. Em relação 
ao modelo analítico, o modelo de simulação não se presta ao tratamento teórico, mas pode ser construído sem necessidade do conhecimento pormenorizado dos princípios elementares inerentes ao sistema real.

Seja por aferição ou modelagem, todavia, um aspecto importante da técnica de avaliação de desempenho utilizada na estimação da capacidade do sistema é sua abrangência sobre o comportamento em condição estática e dinâmica.

\subsection{Avaliação de capacidade estática e capacidade dinâ- mica}

No modo mais comumente empregado na área de sistemas computacionais, a avaliação de desempenho é realizada em regime estacionário, isto é, sob carga de trabalho constante, e depois de findados os efeitos transitórios observados na inicialização do sistema. A avaliação feita dessa forma produz dados para inferir a capacidade estática do sistema. O sistema, inicialmente sem carga, é excitado com a entrada de interesse, e guarda-se até que a saída estabilize-se em seu valor final, quer seja um valor constante, quer seja um valor variável dentro de uma faixa que acomoda tanto a estocasticidade da resposta quanto flutuações periódicas inerentes ao processo. A condição é alcançada passado o assim denominado período de warm up (aquecimento), em que o sistema encontra-se em estado de inicialização. A determinação da capacidade obtém-se estimulando o sistema com cargas gradativamente mais elevadas até atingir seu limite inferior de desempenho aceitável, correspondente à carga estática máxima suportada.

Embora o difundido emprego da avaliação de capacidade estática em sistemas computacionais, é preciso notar que esta abordagem se limita aos resultados que podem ser obtidos pela análise em regime estacionário, excluídos os efeitos do comportamento transitório. Contudo, análise do comportamento nesse período contém outras informações importantes acerca da capacidade do sistema operar em condições reais, em que a carga não é constante. Em regime transiente, a quantidade de recursos necessários para garantir o desempenho especificado pode divergir substancialmente daquela apurada em regime estacionário. Isso ocorre porque, embora durante o regime estacionário possa haver recursos suficientes para a absorção da carga de trabalho estática, durante um período transiente provocado por uma perturbação na carga de trabalho, é possível que a quantidade de recursos exigida seja temporariamente superior àquela prevista para o regime de carga não variável. Isso resulta, por exemplo, de uma abrupta elevação da carga imposta (e.g. súbito aumento de requisições, ou equivalentemente, da parcial de recursos), decorrendo em descumprimento do SLA, ainda que o aumento da carga não exceda a capacidade estática.

Como ilustração, seja um sistema mecânico onde uma mola mantém suspensa uma massa $m$, a lei de Hooke diz que a força $F$ exercida por uma mola ideal é diretamente proporcional e contrária à distensão $x$, de modo que $F=-k . x$, onde $k$ é uma característica própria da mola. Sob a gravidade, e livre de outras interferências, na condição de equilíbrio a mola é esticada da sua 
posição normal, até um alongamento $x_{1}$, ponto em que a força exercida pela mola $-k \cdot x_{1}$ se anula perfeitamente com a força peso $m_{1} . g$ entrando em seu regime estacionário.

O sistema massa-mola pode ser utilizado, em forma de analogia, às informações obtidas pela avaliação de capacidade estática e dinâmica. Nesse sistema, a mola deve suportar uma massa $m$ sem que esta chegue ao nível do solo. Ao pendurar-se uma massa $m_{1}$, a mola se distende até o ponto $x_{1}$, sem tocar o solo, evidenciando a capacidade de suportar a massa. Na avaliação em regime estacionário, aumenta-se a carga gradualmente até que seja encontrada uma massa $m_{3}$ que distenda a mola até o solo, representando, assim, o limite da capacidade estática do sistema. É preciso observar, contudo, que, se no lugar de aumentar gradativamente o valor da massa, um acréscimo súbito for adicionado de forma a massa (carga) passe de $m_{1}$ para $m_{2}<m_{3}$ abruptamente, ainda sem uma análise matemática, intuitivamente pode-se esperar que a mola distenda-se temporariamente além do ponto $x_{2}$, e oscile em torno deste - convergindo eventualmente para o ponto de equilíbrio no caso de um sistema com perda de energia. É possível que essa distensão transiente ultrapasse o nível do solo, extrapolando seu limite no período transitório.

Logo, uma mesma carga que, no regime estacionário é medida como suportável dentro da capacidade estática, no regime transiente pode resultar excessiva em função da velocidade com que é imposta ao sistema. Esse efeito pode ser previsto por técnicas de avaliação de desempenho dinâmica. Em um sistema computacional, embora as propriedades inerciais sejam em geral pouco relevantes em muitas aplicações convencionais, a literatura científica vem sinalizando um maior interesse nesse aspecto, especialmente com o advento de sistemas distribuídos de escala muito grande (Xiong, 2012; Hellerstein et al., 2012). Com essa informação fornecida pela avaliação de capacidade dinâmica é possível prever se um determinado sistema é capaz de suportar perturbações na carga de trabalho, dessa forma, inferindo a necessidade de recursos.

\subsection{Motivação}

A avaliação do regime transiente relativamente é pouco empregada em sistemas computacionais, embora pertença aos fundamentos mais importantes em outras áreas da engenharia e ciência. Em parte, isso pode ser atribuído às características dos sistemas computacionais convencionais. Ao contrário de sistemas mecânicos, elétricos e hidráulicos, que apresentam propriedades inerciais com respeito à transformação de estímulos (entrada) em resposta (saída), sistemas computacionais que representam operações lógicas não evocam a consideração de suas propriedades dinâmicas para propósito de engenharia. Mesmo em sistemas de tempo-real, em que o tempo de resposta à entrada é relevante, apenas métricas de atraso são em geral de interesse. O emergente, e ainda pouco explorado, interesse na análise do comportamento transiente de sistemas computacionais acontece concomitantemente ao aparecimento de aplicações onde a dinâmica dos mecanismos constituintes se torna não negligenciável. É o caso de sistemas distribuídos de larga escala, de grande complexidade e envolvendo a interação pervasiva com sistemas físicos. A escala sem pre- 
cedentes de datacenters que oferecem serviços de computação em nuvem e avanços nas aplicações práticas em computação ubíqua embarcada oferecem bons exemplos (Kannan et al., 2011; Hayes, 2008; Egami et al., 2011). Em tais aplicações, o trânsito das informações de monitoramento de desempenho, algoritmos de tomada de decisão e mecanismos de realocação de recursos possuem tempos de reação, e seus atrasos relativos implicam em uma dinâmica não desprezível.

\subsection{Objetivo}

Este trabalho tem como objetivo a formulação de um método empírico para previsão de sobrecargas transientes em sistemas computacionais. A contribuição almejada é a descrição de um processo prático que possa ser aplicado para determinar a máxima amplitude do impacto sofrido pelo sistema por uma perturbação brusca, e sua duração transitória, até que a saída seja reestabelecida ao valor estacionário final. A aplicação do método deve resultar em um modelo dinâmico simples, possível de ser tratado analiticamente ou numericamente por técnicas clássicas de análise de sistemas lineares invariantes no tempo. O modelo deve descrever a forma como o sistema responde às variações na carga de trabalho e fornecer informações para dimensionamento de recursos. São consideradas perturbações bruscas e de duração prolongada e os resultados são demonstrados e validados por meio da comparação da saída de um protótipo, simulado por um modelo de eventos discretos, e do modelo dinâmico obtido, resolvido em um ambiente de computação numérica. 


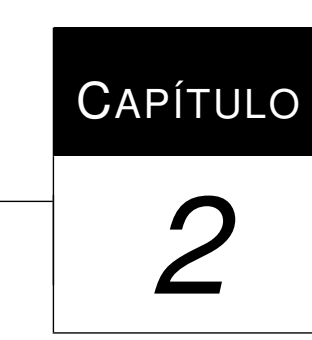

Revisão

Em diversos ramos da engenharia e de várias ciências naturais, técnicas empregadas para análise de desempenho em regime transiente são oriundas de outras áreas como análise de sinais e sistemas e teoria de controle (Ogata, 2010). Na indústria, projetos de sistemas mecânicos, hidráulicos, elétricos fundamentam-se nos seus conceitos há mais de meio século. Esse arcabouço conta com um rico ferramental matemático para modelagem do comportamento estacionário e transiente de sistemas dinâmicos, bem como técnicas específicas para o projeto de estratégias de controle retroalimentado. Não obstante, embora já tenha sido anotado que esse seja um dos aspectos técnicos essenciais à implementação de capacidades auto-adaptativas (Müller et al., 2009) especificadas pela computação autonômica (Horn, 2001; Miller, 2008), representativa contribuição de pesquisa nessa área tem-na tratado sob a perspectiva de arquiteturas de sistemas e modelos de requisitos, ao passo que as técnicas de análise e síntese disponíveis, são ainda pouco exploradas no campo da computação (Hellerstein et al., 2005).

Em seu instrumental analítico e de simulação conta-se com métodos de análise do transiente de sistemas dinâmicos e dos fatores que influenciam perturbações externas e internas na variável de saída, tais como dimensão do impacto, tempo de recuperação e qualidade da estabilização.

A aplicação da teoria de controle em arquiteturas de sistemas computacionais para otimização de desempenho ou confiabilidade é, todavia mais recente e consideravelmente menos explorada que em outros domínios. Tem sido empregada, ainda que menos expressivamente, por exemplo, para clusterização dinâmica de processadores paralelos em problemas de particionamento de recursos, e para formatação de throughput em mecanismos de controle de congestão em redes de comunicação (Abdelzaher et al., 2003; Lu et al., 2001, 1999). Escalonamento retroalimentado ( $f e-$ edback scheduling) é considerado um paradigma de ponta no campo de sistemas de tempo-real (Lu 
et al., 2003; Sha et al., 2004; Abdelzaher et al., 2003; Lu et al., 2001), especialmente em aplicações destinadas a ambientes não-determinísticos. Teoria de controle tem sido também utilizada para controle de admissão adaptativo em arquiteturas multicamadas (Kamra et al., 2004), controle de latência em servidores Web (Henriksson et al., 2004), bem como aplicações em computação em nuvem (Lim et al., 2009), redes de computadores (Xia et al., 2009), computação verde (Wang et al., 2011) (green computing). Considerada ainda uma formação mais específica das engenharias, o reconhecimento da ampla aplicabilidade aos sistemas computacionais, especialmente os de grande escala e complexidade, as oportunidades têm inspirado também iniciativas explicitamente dedicadas a tornar mais acessível a modelagem dinâmica a cientistas da computação por meio de publicações especialmente dirigidas a esse fim (Hellerstein et al., 2005; Zhu et al., 2009; Lunze e Lehmann, 2010; Harjunkoski et al., 2009). O projeto de estratégia de controle para implementar propriedades autonômicas de auto-gerenciamento em resposta a perturbações em tempo de execução, constituem inerentemente exemplos onde o modelo do comportamento transiente é implícito ao atendimento de requisitos de tempo-real. Este é o caso, por exemplo, de trabalhos como Lu et al. (2002, 2006); Abdelzaher et al. (2008); Qin e Wang (2007).

Não obstante a importância de trabalhos seminais como esses mencionados, e de recentes avanços que seguem na mesma linha, em considerável extensão, contribuições são oriundas de pesquisas multidisciplinares e, não infrequentemente, exploradas sob a hipótese de um adequado background em Teoria de Controle. O que nem sempre corresponde ao domínio de conhecimento de especialistas da computação, tanto pela incipiente presença de disciplinas relacionadas nos currículos regulares de formação, quanto pela sua pouca expressão nas abordagens tradicionais da área, quer na academia, quer na indústria. Assim, por vezes percebida como "exótica" na computação, a vasta e admitidamente "pesada" teoria mostra-se pouco encorajadora, especialmente face à pouca compreensão de seu potencial nas aplicações em computação — o que responde pelo grande espaço ainda aberto para a pesquisa nesse campo. Dentre essas possibilidades relativamente pouco exploradas inserem-se as ferramentas de modelagem dinâmica e avaliação de desempenho transiente - foco deste trabalho, particularmente no seu emprego para planejamento de capacidade e política de admissão.

\subsection{Resultados anteriores no grupo de pesquisa}

No Grupo de Sistemas Distribuídos e Programação Concorrente, onde desenvolve este projeto, outros trabalhos de pesquisa anteriores e em andamento exploram essa perspectiva. Nobile (2013) apresenta um estudo que demonstra que as características dinâmicas de um ambiente de computação em nuvem de larga escala tem impacto apreciável no desempenho dos mecanismos de provisão de QoS, e que a modelagem das propriedades de resposta transiente, bem como a aplicação de técnicas de teoria de controle podem ser justificadamente motivadas em projetos de mecanismos de controle realimentado em ambiente de recursos elásticos. $\mathrm{O}$ trabalho de Nobile fornece um roteiro para o projeto de um controle regulatório que gerencia, adaptativamente, máquinas virtuais de um 
datacenter, de modo a manter a taxa de utilização do sistema constante a despeito de variações na demanda. O foco daquele estudo é atuar na velocidade com que novas máquinas virtuais são alocadas ou dispensadas, de modo a atender requisitos temporais.

Tendo o sistema proposto por Nobile como estudo de caso, o método apresentado no presente trabalho de pesquisa é utilizado para modelar o comportamento dinâmico daquele ambiente. A comparação da previsão analítica pela abordagem introduzida com observações experimentais produzidas por simulação é avaliada e discutida nos próximos capítulos.

\subsection{Arquitetura de Nobile}

No trabalho de Nobile (2013) é proposta uma abordagem de implementação de um mecanismo adaptativo de alocação de recursos para uma arquitetura de computação em nuvem, a partir das técnicas de análise e projeto inspiradas em conceitos de controle clássico. O objetivo do mecanismo desenvolvido é regular a taxa de utilização das máquinas virtuais do datacenter.

Tendo a demanda como variável independente (taxa de chegada de requisições e seus custos de processamento), a taxa de utilização pode ser aumentada ou reduzida pelo respectivo aumento ou diminuição do desempenho do datacenter, proporcional ao número de máquinas virtuais. Assim, a estratégia de gerenciamento adaptativo de recursos consiste em iniciar ou desligar máquinas virtuais a fim de manter a taxa de utilização media regulada frente a perturbações externas.

A Figura 2.1 oferece uma representação em alto nível do sistema proposto por Nobile. No diagrama é utilizada a notação convencional: os blocos representam as funções de transferência de cada um dos subsistemas, e as setas indicam seus respectivos sinais de entrada e saída.

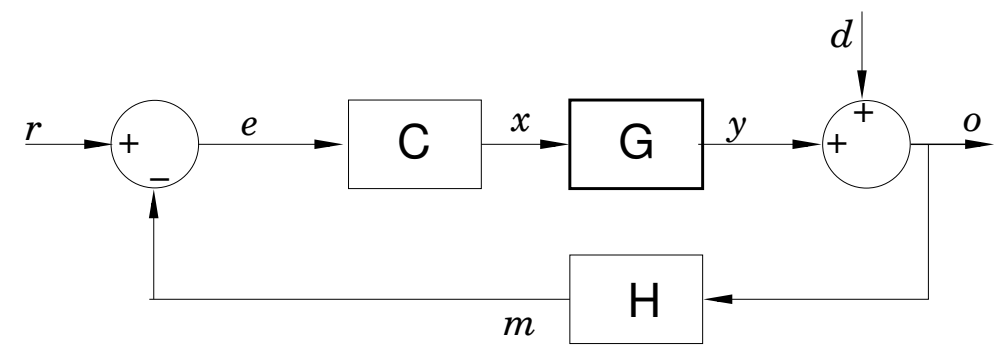

Figura 2.1: Diagrama simplificado da arquitetura de controle de Nobile (Nobile, 2013).

No diagrama o datacenter é representado pelo bloco $G$, sendo a entrada $x$ a quantidade de máquinas virtuais desejadas especificadas e a saída $y$ a taxa de utilização resultante da carga de trabalho imposta ao sistema à entrada. $d$ é uma perturbação imposta à carga causando o aumento ou diminuição na taxa de utilização final $o$. O bloco $H$ é um sensor que mede a taxa de utilização, aplicando-lhe uma média móvel, sendo $m$ seu resultado. O sinal $r$ é o valor da taxa de utilização desejado, sendo $e$ o erro residual de $r-m$. O bloco $C$ é o controlador que utiliza o erro $e$ para gerenciar a quantidade de máquinas virtuais necessárias para manter a taxa de utilização. 
Nesse trabalho foram empregadas técnicas para identificação do sistema além de um projeto de controlador considerando requisitos dinâmicos, como: máximo overshoot (sobrepassagem) e máximo settling time (tempo de assentamento) devido a uma perturbação. O controlador desenvolvido demonstrou maior eficiência em relação às abordagens baseadas em técnicas ad hoc. Ainda mostrou que mesmo sistemas de larga escala e alta complexidade podem ser aproximados por modelos matemáticos simples.

Os resultados são avaliados mediante simulação com o auxílio da ferramenta CloudSim (Buyya et al., 2009). CloudSim é um framework robusto, amplamente utilizado e referenciado na literatura. O software permite que ambientes de nuvem de larga escala compostos de vários nós sejam avaliados com a implementação simples de um programa de simulação. Os resultados obtidos utilizando CloudSim mostraram-se coerentes com aqueles previstos pela modelagem. 
Formalmente, um sistema dinâmico é aquele cuja transformação entrada-saída não ocorre em tempo zero, mas é sujeita a uma inércia advinda dos processos físicos associados. Sistemas mecânicos, térmicos, elétricos, para citar alguns exemplos, possuem uma inércia intrínseca que atrasa o efeito que uma entrada terá na saída. Em sistemas computacionais esses efeitos decorrem da atuação de buffers e tempo de instanciação de novos recursos. Essa propriedade dinâmica tem como consequência comportamentos diversos que incluem retardo no tempo de resposta e possíveis oscilações e definem a estabilidade do sistema. Em muitos sistemas computacionais convencionais, há pouco interesse em modelar o comportamento dinâmico, dado que, por um lado a preocupação não concerne a operações lógicas, e por outro, mesmo quando existem requisitos de tempo real, em geral apenas atrasos são relevantes.

Com o aumento da escala e complexidade de sistemas computacionais distribuídos, propriedades dinâmicas não desprezíveis, emergem da combinação dos vários elementos que o compõe, de modo que uma perturbação na carga de trabalho pode resultar em uma resposta transiente com impacto relevante no desempenho. Um exemplo representativo é o caso das infraestruturas de datacenters em aplicações de cloud computing cuja escala de implantação ressalta os efeitos inerciais combinados. Nesse caso, a modelagem de sistemas computacionais como sistemas dinâmicos torna-se potencialmente importante.

Para a realização dos estudos deste trabalho, considera-se a representação do sistema por meio de simulação e sua respectiva análise através de softwares que implementam métodos computacionais que solucionam os modelos dinâmicos a serem empregados. Desse procedimento resultam equações analíticas que representam o comportamento do sistema com respeito ao formato da saída, dada uma determinada entrada. A avaliação da qualidade desse modelo, no presente estudo, 
é feita comparando-se a previsão com os resultados gerados pelo simulador específico CloudSim (Buyya et al., 2009), respaldado na literatura científica e que reproduz, por meio de modelos de filas e eventos discretos, o comportamento de uma infraestrutura de computação em nuvem.

\subsection{Resultados Preliminares do Estudo}

A formulação da descrição de um sistema é designada pelo conceito de modelagem. O processo consiste basicamente em expressar matematicamente o comportamento de interesse de modo que as características do sistema estejam suficientemente representadas. Quando a relação entradasaída tem um comportamento dinâmico (i.e. a saída depende da entrada, mais do estado atual do sistema, caracterizando portanto uma memória “inercial”), o modelo corresponde a equações diferenciais. No caso de sistema que apresentam propriedades lineares (sinais de entrada superpostos são combinados linearmente na saída), e cujos parâmetros são fixos (não alteram-se ao longo do processo), o sistema pode ser representado por um modelo linear invariante no tempo (LTI). Essa condição tem uma razão prática em Engenhria, pois admite soluções analíticas viáveis.

No caso de sistemas discretos, a formulação é por meio de equações de diferenças que representam como a saída varia em função do tempo. Na teoria clássica de sistemas LTI de única entrada e única saída, é conveniente a representação das equações de diferença na sua forma equivalente no domínio da frequência, mediante a transformada $\mathrm{Z}$, análoga à transformada de Laplace para o domíno contínuo. Nessa forma, cada bloco do sistema da Figura 2.1 denota uma função de transferência que converte a entrada na saída, tendo em conta os efeitos inerciais. As funções de transferências têm a conveniente propriedade de poderem ser multiplicadas algebricamente quando em arranjos sequenciais, de modo que, no diagrama, a relação $y=g(c(x))$, no domínio do tempo, se torna $Y=F G . X$.

A forma mais direta de obter-se um modelo expressivo é a partir de métodos analíticos no qual princípios básicos ( $i$. e l leis da Física, Elétrica ou área da Ciência em que se encontra o sistema em questão) são considerados para que um modelo seja encontrado, o que resulta em uma equação diferencial para um sistema contínuo ou uma equação de diferença para sistemas discretos. A modelagem analítica por princípios elementares é muito útil quando o conhecimento interno de um sistema pode ser convenientemente suposto, entendido e então modelado, o que é muito comum para alguns sistemas mecânicos ou elétricos, mas que pode ser de difícil aplicação em sistemas computacionais.

Quando o sistema em questão é computacional, algumas nuances devem ser consideradas. Uma delas, é que sistemas computacionais são bastante abstratos, o que significa que supor como se dá o comportamento interno de um sistema de computação não é uma tarefa simples, muitas vezes inviabilizando a utilização de técnicas dedutivas de modelagem. É interessante destacar também que quanto mais simples for o sistema ( $i$. e. a dinâmica capturada no modelo) mais fácil é o processo de modelagem, todavia, sistemas reais não são simples. Para casos assim, é possível que 
aproximações e ponderações sejam feitas de modo a simplificar o sistema e facilitar a extração apenas do comportamento essencial do mesmo.

A modelagem de sistemas complexos pode ser feita por meio de outras abordagens nas quais o conhecimento tácito do comportamento interno não seja necessário. Técnicas assim são comumente referenciadas como de caixa preta ${ }^{1}$ e Hellerstein et al. (2004) propôs um fluxograma para aplicação de técnicas desse tipo que é ilustrado na Figura 3.1.

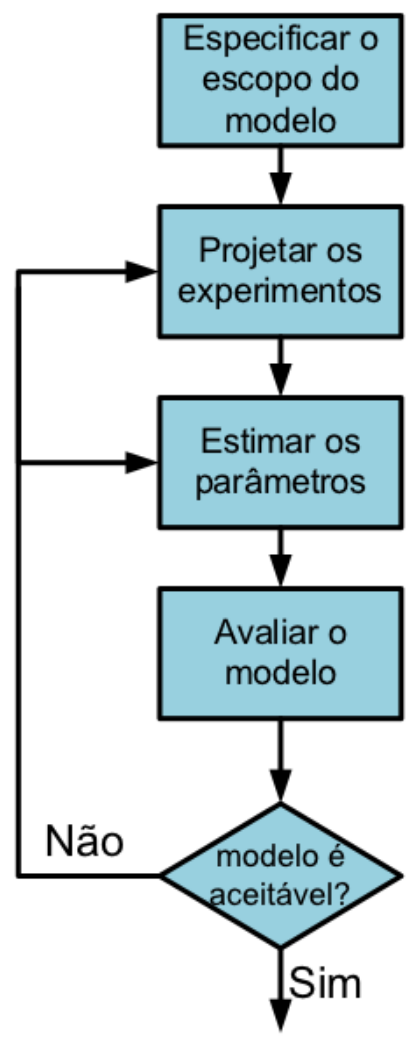

Fim

Figura 3.1: Etapas para modelagem de caixa preta de um sistema dinâmico.

A abrangência e os limites dos modelos são definidos numa primeira etapa conhecida por escopo do modelo. Nessa etapa são escolhidas as variáveis de interesse (i. e. entrada e saída) que serão usadas para extrair o modelo, além de definir-se quão representativo será o mesmo. Quanto maior a representatividade, maior será a adequação do modelo ao sistema real. Se por um lado pode parecer adequado que quanto mais representativo melhor é o modelo, por outro lado corre-se o risco de um superajustamento que faz com que o modelo seja, de fato, representativo apenas para o conjunto de dados usados no processo de modelagem. Nessa etapa é necessário encontrar um equilíbrio entre representatividade e suficiência para evitar desarranjos no ajuste do modelo ao sistema real.

Nessa fase também são definidas qual será a entrada submetida e qual a saída esperada no processo de modelagem. Tanto a entrada quanto a saída devem representar a atuação real do sistema

\footnotetext{
${ }^{1}$ Black-box system identification.
} 
que se quer modelar. É importante que o comportamento expresso pela aplicação das entradas e posterior coleta das saídas aplicadas ao sistema, consiga excitá-lo dentro de suas condições normais de operação.

Tendo o escopo bem definido, o planejamento de experimentos deve deixar claro qual será o ponto ou intervalo de operação em que se deseja reconhecer a dinâmica do sistema. O planejamento correto resultará em um conjunto de entradas suficiente para exprimir a dinâmica que se deseja observar. Posteriormente, o sistema deve ser submetido aos dados às entradas escolhidas e a resposta deve ser coletada para que uma relação de transformação seja encontrada. Uma forma é utilizar-se de modelos autorregressivos que representem a transformação de entrada em saídas e então utilizar os dados coletados para estimar os parâmetros necessários.

Uma técnica bastante útil foi descrita por Parekh et al. (2002) que considera a utilização de modelos autorregressivos para identificar sistemas invariantes no tempo que obedeçam aos princípios de causalidade e superposição. Enquanto o princípio de causalidade diz que uma resposta do sistema deve depender apenas das entradas anteriores à mesma, a superposição diz que se uma resposta do sistema é fruto da aplicação da combinação de duas entradas, então a mesma resposta pode ser obtida da soma das respostas individuais resultantes da aplicação individual das entradas consideradas. Esse princípio é especialmente útil para definição de sistemas lineares: sistemas lineares são aqueles no qual o princípio de superposição é observado.

\subsection{Estudo de caso: arquitetura de Nobile}

Um exemplo da aplicação de identificação da dinâmica de um sistema computacional usando um método de caixa preta que aproxima o comportamento para um modelo autorregressivo de primeira ordem pode ser encontrado em (Nobile, 2013). Nesse caso foi considerada como variável de entrada a quantidade de instâncias de máquinas virtuais que um sistema de computação em nuvem necessitaria para o atendimento de uma demanda gerada pelo envio de requisições, e como resposta foi observada a taxa de utilização desse conjunto de máquinas virtuais.

A fim de identificar a relação de transformação da aplicação das entradas na saída, o sistema foi submetido a uma perturbação dentro de um intervalo de operação na variável de entrada, na forma de um degrau para baixo, como mostra a Figura 3.2, sendo a resposta observada ilustrada na Figura 3.3. A simulação considerou inicialmente instâncias de máquinas virtuais que resultavam numa resposta estacionária com taxa de utilização de aproximadamente 54\%. O degrau foi provocado na origem dos tempos fazendo com que a quantidade de instâncias passasse a 14, aumentando a taxa de utilização para 99\% aproximadamente após alguns instantes.

Abstraindo as oscilações e variações provocadas pela existência de estocasticidades intrínsecas do sistema, da carga submetida e outras envolvidas no experimento realizado, a Figura 3.3 mostra claramente a dinâmica aparente na resposta quando há variação da entrada. Essa dinâmica pode 


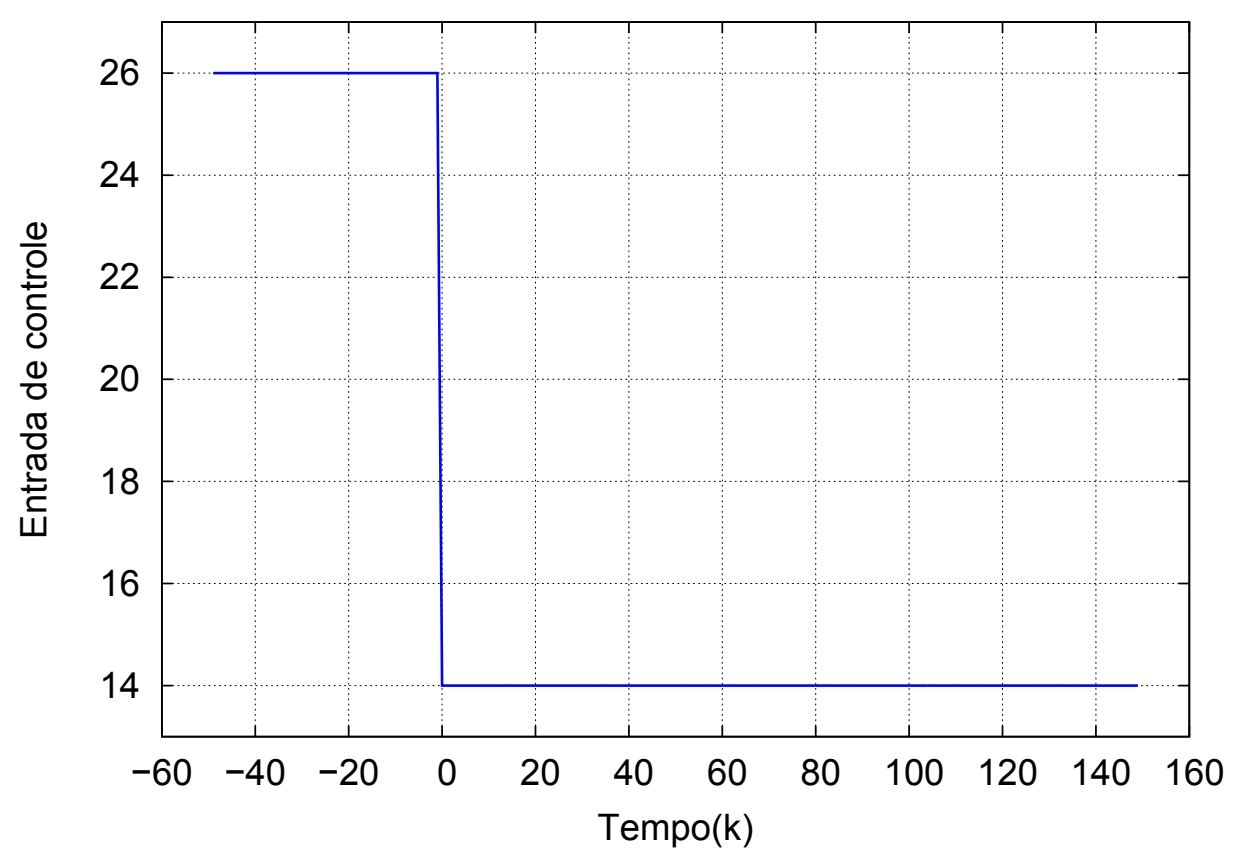

Figura 3.2: Intervalo e variação de entradas aplicados à modelagem do sistema

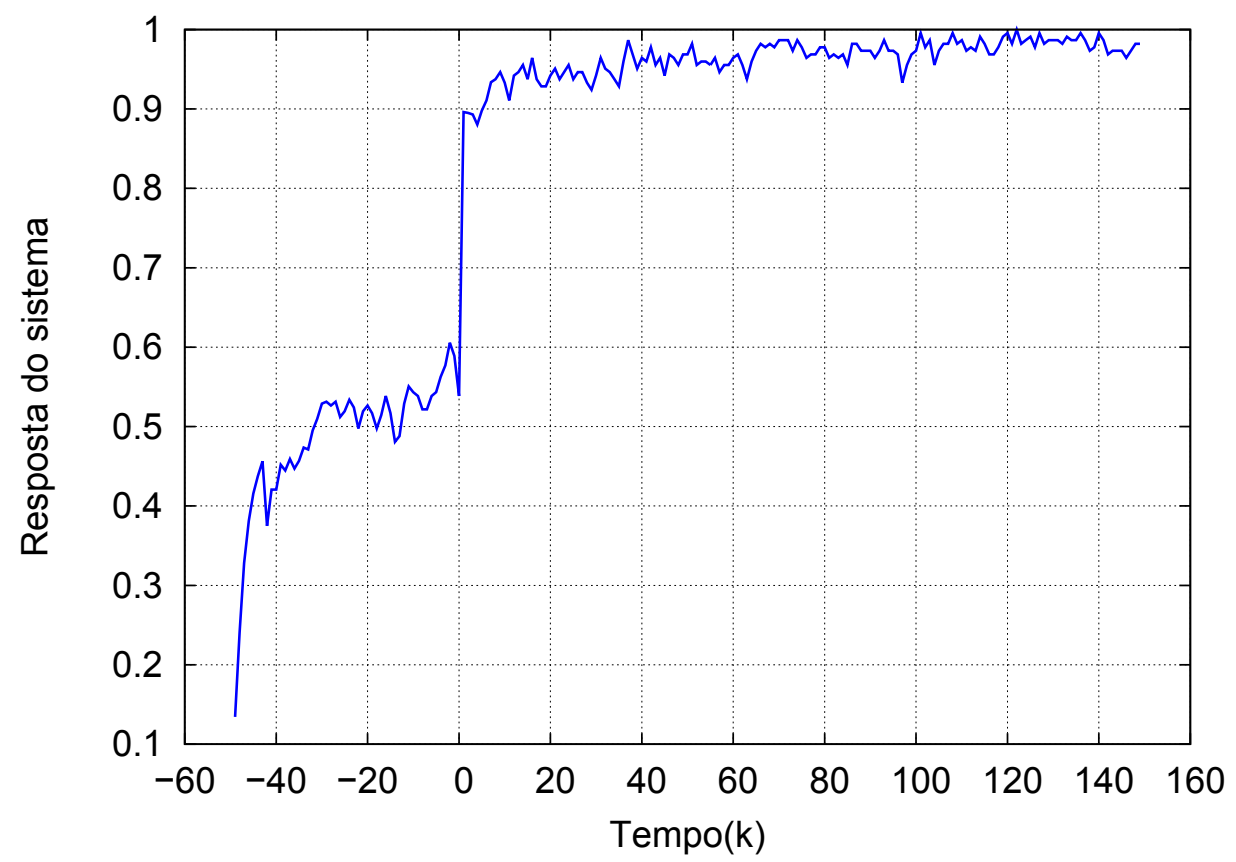

Figura 3.3: Saída observada do sistema em resposta à variação da entrada

ser expressada por um modelo autorregressivo de primeira ordem ${ }^{2}$, como o mostrado pela equação 3.1, em que $\hat{y}(k+1)$ é o valor da saída previsto no instante $k+1$ com base nos valores da entrada aplicada ao sistema em $k, u(k)$, e na saída medida ou prevista em $k, y(k)$ ou $\hat{y}(k)$. Outras ordens podem ser consideradas caso modelos de ordem baixa sejam pouco representativos.

\footnotetext{
${ }^{2}$ Pelo princípio da parcimônia, onde há preferência pela explicação mais simples, faz sentido considerar o modelo mais simples capaz de explicar os resultados.
} 


$$
\hat{y}(k+1)=a y(k)+b u(k)
$$

A aplicação do método de mínimos quadrados nos valores das Figuras 3.2 e 3.3, descontado o warm up e transladado a saída inicial para a referência zero, resultou no seguinte modelo com os parâmetros $a$ e $b$ estimados, como mostra a equação 3.2.

$$
\hat{y}(k+1)=0.3825 \hat{y}(k)-0.02251 u(k)
$$

A adequação do modelo pode ser comprovada visualmente e numericamente, como mostram as Figuras 3.4(a) e 3.4(b), e numericamente, como mostra a Tabela 3.1, pela aplicação dos testes estatísticos $R^{2}$, RMSE e coeficiente de correlação. O valor de $R^{2}$ varia entre 0 e 1 , quanto mais próximo de 1, melhor o ajuste do modelo. Seu valor é obtido pela equação 3.3.

$$
\sqrt{\frac{1}{n} \sum_{i=1}^{n}[y(i+1)-\hat{y}(i+1)]^{2}}
$$

A raiz quadrada da média do erro (RMSE), equação 3.4, indica o desvio padrão dos erros, quanto mais próximo de zero, melhor o ajuste.

$$
R^{2}=1-\frac{\operatorname{var}(y-\hat{y})}{\operatorname{var}(y)}
$$

Por fim, o coeficiente de correlação, utilizado para averiguar a qualidade do ajuste do método dos mínimos quadrado em relação aos dados utilizados. Indica a correlação entre a entrada e saída prevista e sua intensidade. Seus valores variam de -1 a 1 , onde 0 não há correlação, -1 indica forte correção negativa e 1 correção positiva. Calculado pela equação 3.5.

$$
r=\frac{\sum_{i=1}^{n}(x i-\bar{x})(y i-\bar{y})}{\sqrt{\sum_{i=1}^{n}(x i-\bar{x})^{2}} \sqrt{\sum_{i=1}^{n}(y i-\bar{y})^{2}}}
$$

Na Figura 3.4(a) a resposta observada é ilustrada com aquela obtida da aplicação do mesmo conjunto de entradas ao modelo encontrado. É possível verificar que há uma semelhança visual muito grande entre as curvas de ambas as saídas, o que sugere a adequação do modelo encontrado ao sistema real. Também é possível verificar que há um acompanhamento entre as duas saídas, mas não uma sobreposição completa, o que também indica um ajustamento nem muito frágil, nem muito rígido do modelo.

Já na Figura 3.4(b), ambas as saídas são confrontadas utilizando como coordenadas o valor da saída observada e da saída resultante do modelo para um mesmo intervalo de amostra (i. e. um intervalo de tempo discreto). Na Figura percebe-se que os pontos resultantes do confrontamento das respostas está muito próximo, mas não exatamente sobre o segmento de reta diagonal onde 


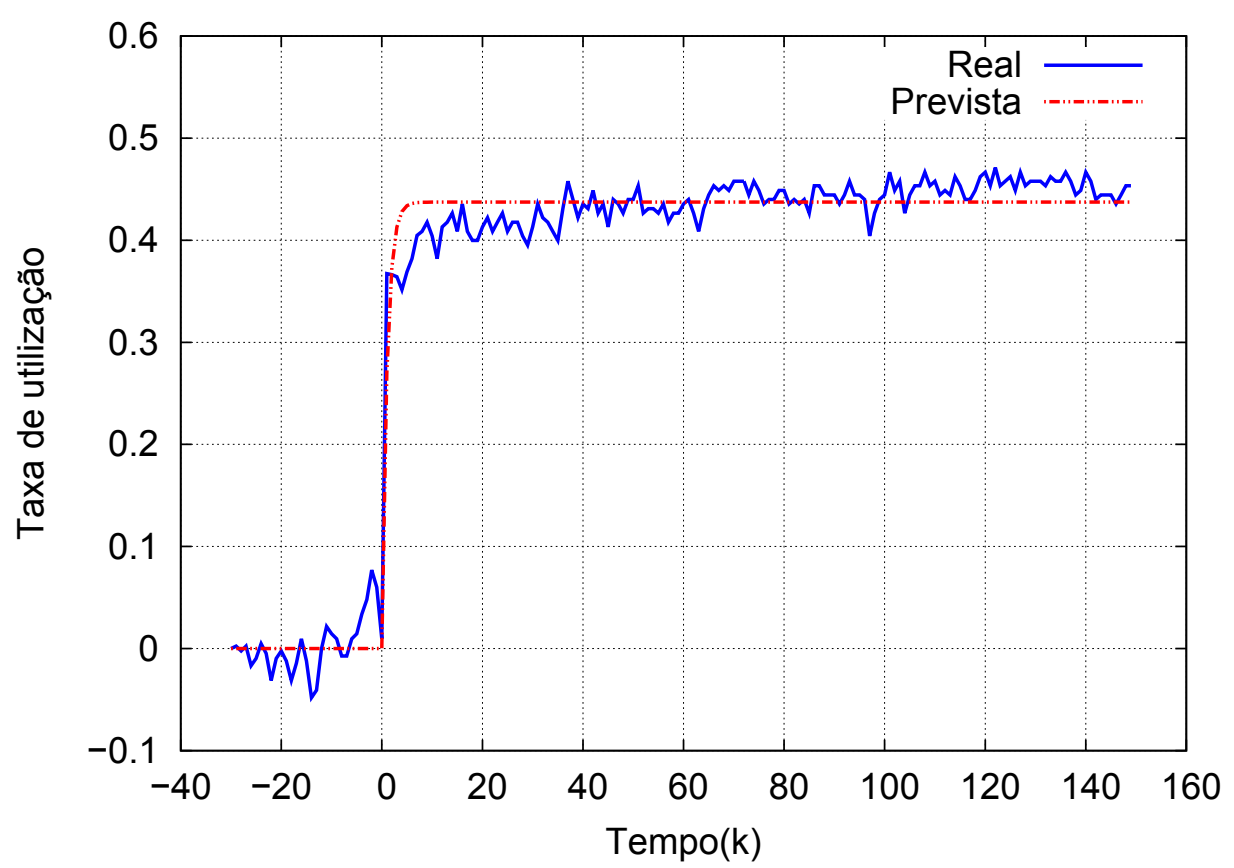

(a)

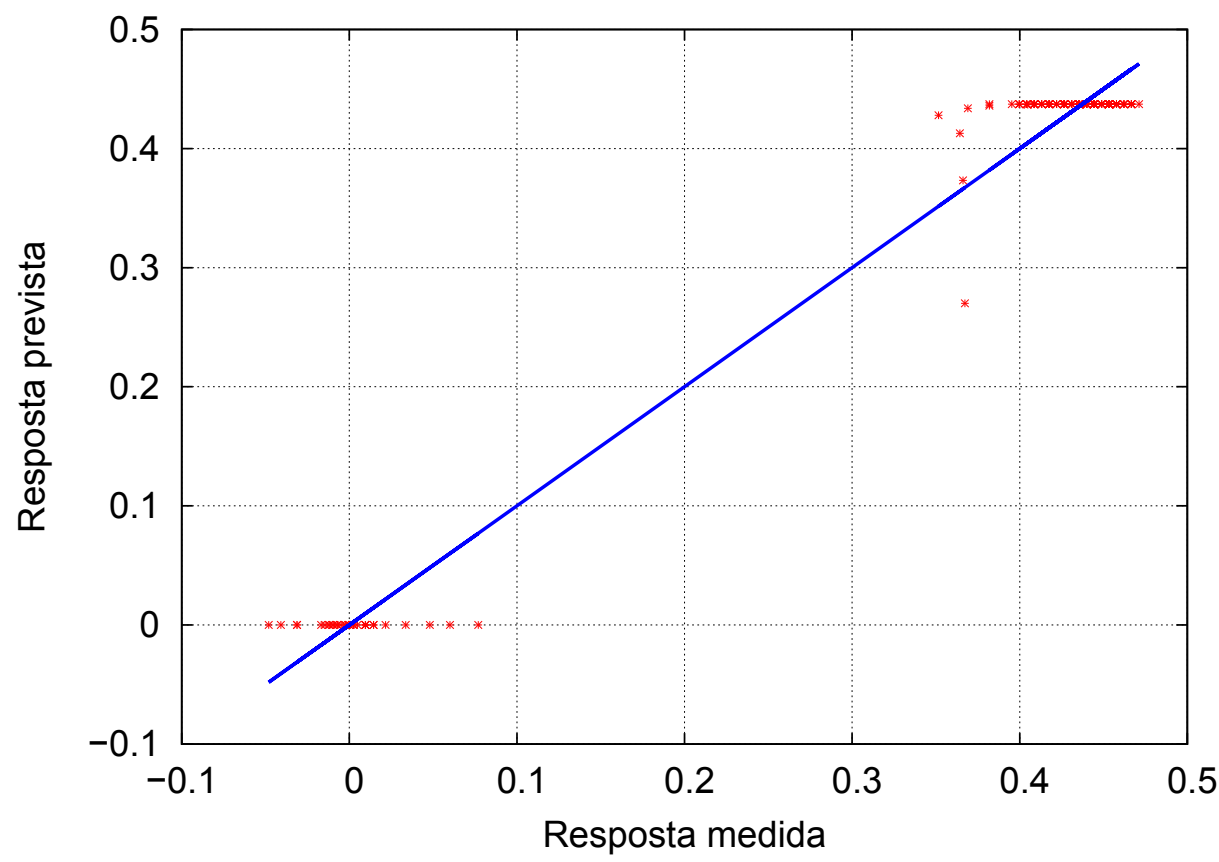

(b)

Figura 3.4: Comparação da resposta real medida com a resposta prevista pelo modelo

$\hat{y}(k)=y(k)$ serve de referência à comparação dos valores, dando novamente evidências de um bom ajustamento.

A comprovação de que o modelo encontrado é adequado ao sistema real pode também ser obtida por métodos numéricos como os mostrados na Tabela 3.1. Nesse caso, os valores próximos a zero da raiz quadrada da média do erro (RMSE), a correlação entre as entradas e saída prevista 
próximo de -1 , além do valor da variabilidade $R^{2}$ próximo de um, reforçam a hipótese de bom ajustamento dado pelos testes visuais.

Tabela 3.1: Valores de $R^{2}, R M S E$ e correlação entre entrada e erro residual para o modelo

\begin{tabular}{lc}
\multicolumn{2}{c}{ estimado. } \\
\hline \hline$R^{2}$ & Valor calculado \\
$R M S E$ & 0.98081 \\
Correlação & 0.02289 \\
\hline
\end{tabular}

Confirmada a adequação do modelo encontrado ao sistema real, é possível afirmar sobre suas características dinâmicas com base nesse modelo. É possível, inclusive, que o comportamento de variáveis de interesse sejam extraídos ao longo do tempo exclusivamente pela análise do modelo, principalmente aquelas apresentadas em períodos transientes como o tempo necessário para que a resposta do sistema volte a estacionar sobre um valor de interesse. Todavia, algumas afirmações são dificilmente extraídas no domínio do tempo e, sendo assim, uma boa estratégia é usar de transformações para domínios em que essas afirmações sejam mais facilmente verificadas, por exemplo, para o domínio $Z$ no caso de sistemas discretos.

O roteiro de trabalho inicia-se com as técnicas clássicas de modelagem de sistemas dinâmicos, considerando-se aproximações para sistemas lineares invariantes no tempo. O sistema-exemplo estudado é modelado por um modelo de eventos discretos e, a partir de técnicas convencionais de injeção de entradas e análise das saídas, aproximações para seu modelo dinâmico analítico. Posteriormente esse modelo é validado comparando-se as previsões das respostas transientes por ele fornecidas em confronto à simulação discreta. Avaliações de resultados são realizadas mediante a comparação entre as predições do modelo dinâmico e os valores experimentais simulados no modelo discreto. 
No presente estudo, a arquitetura de Nobile (2013), esquematizada na Figura 2.1, é tomada como uma caixa preta, como na Figura 4.1. Objetiva-se obter um modelo que informe como o número de máquinas virtuais, adaptativamente instanciadas e desativadas pelo gerenciador adaptativo de recursos elásticos, varia em função de um aumento da carga de trabalho. O sistema informa o número de máquinas virtuais de duas formas:

- quantidade de máquinas virtuais atualmente em execução (operacionais).

- quantidade de máquinas virtuais desejadas pelo controlador para suportar a carga de trabalho (operacionais + em inicialização).

Considerando que a cobrança por máquinas virtual inicia-se no processo de inicialização (boot) (Amazon, 2014), a variável de saída do controlador permite evitar a cobrança desnecessária durante o overshoot.

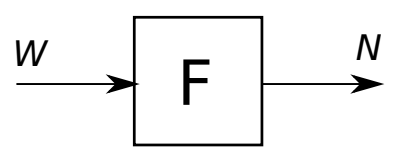

Figura 4.1: Diagrama do sistema.

Evidentemente há uma relação proporcional entre as duas variáveis (pela ação do controlador de Nobile), número de máquinas virtuais e carga de trabalho, mas este estudo demonstra que essa relação é dinâmica e que existem componentes transientes. Isso significa que, se o sistema opera inicialmente com um número médio de máquinas, uma perturbação na entrada pode produzir um 
pico transitório nesse valor, que pode perdurar até que as novas máquinas absorvam a demanda e levem a saída a um novo patamar estacionário.

\subsection{Abordagem e hipóteses}

Sinal é um termo utilizado para se referir ao valor de uma função no tempo (Ogata, 2009); no presente estudo, descrevem-se, portanto, sinais de entrada e de saída. Sua relação de transformação, assumindo a hipótese de que é válida uma aproximação por um sistema linear invariante no tempo (LTI), pode ser descrita por uma função de transferência.

Assumindo, também, as hipóteses de a) uma condição de estabilidade e b) uma dinâmica de ordem maior que unitária (Nobile, 2013), uma perturbação brusca e duradoura (na forma de um degrau) no sinal de entrada deve resultar em uma correspondente perturbação transiente no sinal de saída na forma de um pico, o qual é esperado que se dissipe conforme a saída se estabilize em torno do valor estacionário final. Esse valor máximo em que a saída supera o valor estacionário final é designado sobrepassagem (ou "overshoot"), e sua duração, tempo de assentamento ("settling time").

Pertence ao escopo do método investigado a proposta de abordar a característica estocástica dos sinais de entrada e saída como sinais determinísticos. Para isso, sob carga de trabalho constante, consideram-se a entrada e saída como processos estocásticos estacionários, e como sinais os seus valores médios calculados em uma janela. Esse tratamento permite que um modelo dinâmico de ordem reduzida, por exemplo de segunda ordem, possa ser utilizado como aproximação para os valores médios.

Nos estudos, o sistema de referência é um protótipo simulado no ambiente CloudSim (Buyya et al., 2009), que utiliza modelos abstratos de recursos computacionais resolvidos por simulação de eventos discretos. O comportamento apresentado pelo sistema de referência deve ser reproduzido pelo modelo analítico resultante do método proposto. Esse modelo é de um sistema dinâmico descrito por uma função de transferência. A avaliação da qualidade do modelo analítico é feita comparando-se os sinais de saída do sistema referência (simulação no CloudSim) e do modelo dinâmico ajustado, resolvido no ambiente de computação numérica. Para esse último passo, o software Octave (Octave community, 2014) foi utilizado. Ambas as ferramentas são distribuídas com licenças de software livre.

A carga de trabalho é considerada um processo de Poisson, com tempo médio entre eventos $\mu$ e tempo médio de processamento $\lambda$, ambos dados por distribuições de probabilidade exponenciais. A hipótese da sucessão de eventos sem memória é utilizada em outros trabalhos na literatura com os quais se deseja comparar os resultados (incluindo-se o estudo de Nobile (2013)). Uma vez que o sinal de entrada é a carga média $W=\lambda / \mu$, a forma da distribuição especificamente não é relevante para o tipo de análise feita neste estudo. 
A combinação das duas distribuições exponenciais que parametrizam a carga de trabalho produz um valor $W$ estocástico com grandes variações na forma de um ruído branco de alta frequência. $\mathrm{O}$ fato de a frequência de amostragem ser bastante inferior à frequência do ruído estocástico (adiante, mensurada no gráfico da Figura 4.7) não compromete o resultado, dado que busca-se reconstruir valores médios estacionários e não o ruído. 


\subsection{Procedimento experimental}

A implementação envolveu o uso de dois ambientes: (1) o de geração de dados empíricos, que resolveu o modelo em questão e serviu como uma bancada de testes que simulava o comportamento de um sistema real. Pelo fato de ser implementado em forma de simulação, ajustes, alterações e adequações foram desempenhadas de forma rápida; e (2) aquele para o tratamento dos dados gerados, que utilizou de ferramenta específica para esse fim, diferentemente se tivesse sido adotada uma linguagem de programação de propósito geral (como $\mathrm{C}, \mathrm{C}++$, Java etc.). Isso proporcionou um foco maior nas informações contidas nos dados do que na implementação para sua extração.

Uma vez definido o modelo a ser estudado, sua respectiva solução foi implementada no simulador de computação em nuvem CloudSim (Buyya et al., 2009). Parte da implementação foi aproveitada do código produzido por (Nobile, 2013). Especificamente, adicionou-se variáveis de interesse tanto de entrada, variáveis que provocam um distúrbio no sistema (exemplo: tamanho da carga de trabalho dentro do intervalo de amostragem). Ao adicionar novas variáveis, um código capaz de extrair seus valores foi, consequentemente, adicionado à versão original de Nobile.

Os recursos computacionais simulados tratam de um centro de dados (datacenter), em que máquinas virtuais (Virtual Machine - VM) podem ser alocadas sobre demanda. A partir do momento que novas requisições são geradas, em um dado instante de tempo durante a simulação, elas são alocadas em VMs para processamento. Um monitor faz a checagem do estado das VMs e repassa essa informação a um componente que faz o dimensionamento da potência computacional utilizada para atender as requisições que chegam ao sistema.

Os valores produzidos pela simulação foram adicionados a arquivos no formato de valores separados por identificador (comma separated values - CSV).

O dados produzidos pela simulação foram processados pelo Octave ${ }^{1}$, de forma que todos os arquivos CSV gerados foram carregados na interface. Os dados carregados foram mapeados como matrizes. O pacote control do Octave para processamento de sinais e controle foi utilizado. Cada coluna da matriz de dados carregados corresponde aos resultados de uma variável no tempo e seu valor é interpretado como um sinal.

Os dados produzidos são compostos por vários arquivos, referentes a replicações de experimentos. O cálculo de média e processamento de cálculos estatísticos foram feitos diretamente nas matrizes, de forma correta e simples, uma vez que essas funções já estão implementadas.

Uma vez que o tratamento estatístico foi realizado, funções específicas para a estimação de parâmetros dos modelos que poderiam representar o sistema foram invocadas. Existem estruturas de dados específicas como i ddat a que agrega os valores entrada e saída para serem passadas para a função arx, que implementa o método dos mínimos quadrados como algoritmo de estimação.

No contexto deste trabalho há duas unidades relacionadas a referências métricas ao tempo.

\footnotetext{
${ }^{1}$ http://octave.org
} 
1. Unidade de Tempo da Simulação — $\mathrm{rtu}^{2}$. Referente ao tempo da simulação, às unidades de tempo relacionadas aos eventos que correspondem a fatos que ocorrem durante a execução do simulador. São exemplos que se referem a esse tempo, a chegada de uma requisição ao sistema no instante de tempo $25 \mathrm{rtu}$; sua respectiva alocação em uma determinada VM no instante 37.8 rtu; sua saída em 43.2rtu. Vale mencionar que sua incidência é eventual e foi relacionada a uma distribuição de probabilidade definida nos experimentos.

2. Unidade de Tempo de Amostragem - stu ${ }^{3}$. Essa unidade refere-se ao intervalo entre as amostras das variáveis de interesse. O monitor serviu como um coletor periódico dos valores e seu período é referenciado pela variável $k$.

Assim, eventos são relacionados à unidade métrica $r t u$ e dados coletados à stu.

A simulação não instancia uma aplicação em específico. Se esse fosse o caso, seria possível especificar uma unidade de tempo significativa (por exemplo, segundos, minutos, horas etc.), tanto para o caso rtu quanto para stu. A implementação faz referência a uma abstração de um serviço que recebe requisições e faz o seu processamento. Portanto, é conveniente deixar indefinida a unidade a fim de que, no caso de uma instância, seja possível substituir rtu pela aplicação necessária.

\subsubsection{Experimentos para coleta de dados}

O objetivo dos experimentos foi gerar dados que continham o comportamento dinâmico do sistema. Para isso, precisou ser feita a escolha de uma variável de entrada capaz de excitar o sistema, ou seja, gerar um impacto em uma variável saída.

A variável de entrada foi denominada carga de trabalho $W$, definida por

$$
W=\frac{\mu}{\lambda} .
$$

Em que $\mu$ é o tempo médio de processamento e $\lambda$ é o tempo médio entre chegadas de requisições, ambos os tempos são medidos em rtu. A variável de saída considerada foi a quantidade de Máquinas Virtuais $N$ disponíveis para atender as requisições presentes no sistema.

Pode-se relacionar diretamente a entrada com a saída, porque, ao aumentar o valor de $W$, mais VMs serão necessárias para tratar requisições pendentes, de acordo com a lógica de uma aplicação em nuvem auto escalável. Em outras palavras, ao submeter o sistema a uma carga de trabalho maior, existe um mecanismo capaz de adaptar sua capacidade para atender aquela carga seguindo algum critério especificado.

Para que os dados produzidos pelos experimentos tivessem informações sobre seu comportamento dinâmico, optou-se por escolher uma perturbação na variável de entrada na forma de um degrau. Partindo do princípio que o sistema é linear e invariante no tempo, o sinal degrau com

\footnotetext{
${ }^{2}$ Real-time unit.

${ }^{3}$ Sample time unit.
} 
sua amplitude devidamente ajustada acarreta uma mudança brusca na carga imposta ao sistema. Percebe-se isso ao analisar que em um instante de tempo $\alpha$ a carga de trabalho passa do patamar "atual" para outro diferente no instante $\alpha+1$. O resultado esperado é que, desde que haja correlação entre entrada e saída, a variável de saída seja levada ao novo patamar. Por questões que envolvem o preenchimento de buffers, espera em fila, realocação de recursos etc., o tempo de reação tende a ser apreciável e a seguir um padrão, apresentando um comportamento dinâmico observável. Conforme descrito por (Aguirre, 2007; Ogata, 2009), a excitação de um sistema com um sinal do tipo degrau é amplamente utilizada e adequada para produção de dados para identificação de modelos de função de transferência.

Como o intervalo de amostragem será maior que o valor de 1stu, é interessante explicar como será calculado o valor a ser coletado. Todos os valores gerados pela simulação, de frequência eventual, foram considerados, pois, entre o intervalo de amostragens, uma quantidade diferente de eventos pode acontecer. A solução adotada foi calcular o valor médio ocorrido naquele período e esse ser o valor considerado como amostrados.

Os experimentos consistem em aplicar uma carga de trabalho com intensidade relativa 7.0 e, no instante de tempo $1000 \mathrm{stu}$, alterar seu valor para 14.0, permanecendo neste valor até o final da simulação no instante de tempo 1999stu - i.e., um degrau de amplitude 7.0 ou o dobro da carga inicial. Os valores das variáveis foram amostradas periodicamente com frequência $\tau=10 \mathrm{rtu}$.

\subsection{Resposta ao degrau}

Buscando identificar a relação de transformação da entrada na saída, o sistema foi submetido a uma variação do tipo degrau. No instante de tempo da amostra $k=1000$ stu a média da carga de trabalho sofreu um aumento abrupto de $W=7$ para $W=14$, sendo demonstrado no gráfico 4.2. A resposta do sistema à entrada e sua reação ao degrau pode ser observado no gráfico 4.3. Embora seja visualmente perceptível o impacto causado pelo degrau no número de máquinas virtuais, a dispersão da carga de trabalho é grande gerando um ruído maior que o próprio degrau, o que dificulta seu discernimento.

Ademais, um modelo LTI de baixa ordem não é suficiente para se ajustar a estocasticidade apresentada pela entrada. Para se adequar aos requisitos de um modelo de baixa ordem, é necessário diminuir a estocasticidade do sistema. Um método para atender a essa meta é através da replicação do experimento utilizando diferentes sequências aleatórias.

\subsection{Replicações}

A replicação do experimento ajuda a diminuir a estocasticidade. Sendo o número de máquinas virtuais e a carga de trabalho uma média constante definidas por uma distribuição, para cada replicação uma semente diferente, gerada aleatoriamente, é fornecida. Cada semente garante que 


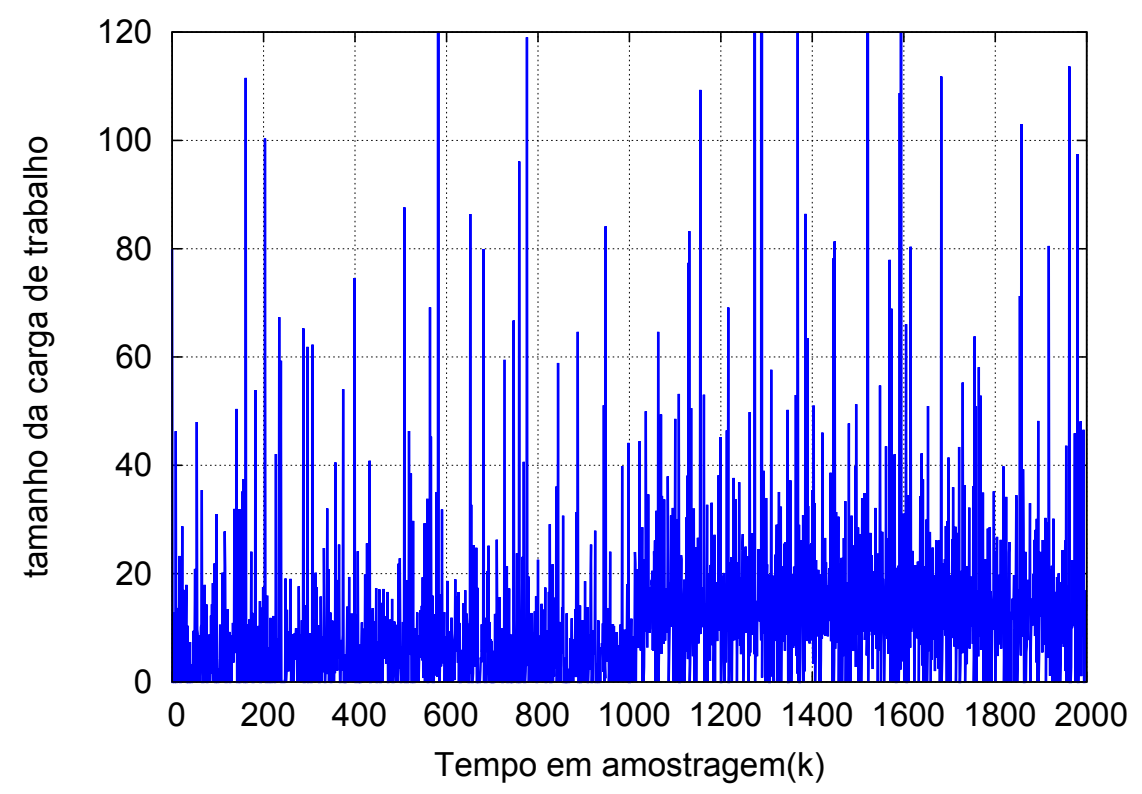

Figura 4.2: Carga de trabalho $(W)$ de uma única replicação

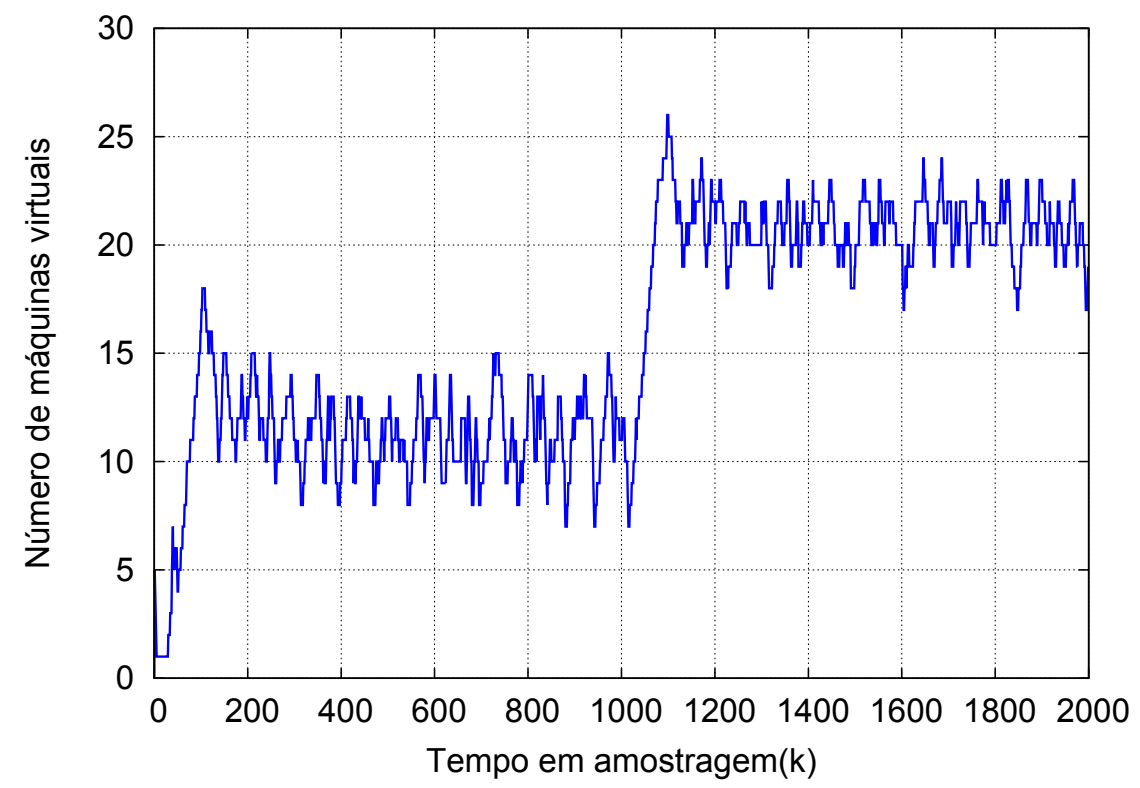

Figura 4.3: Número de máquinas virtuais $(N)$ de uma única replicação

as replicações sejam distintas, com valores de carga de trabalho diferentes, mas constantes sobre a mesma média. Sendo a entrada uma média estacionária, o aumento do número de replicações permite diminuir o ruído estocástico, pois espera-se que a média das replicações tenda para a média definida no experimento. Dessa forma, neste experimento, aumentou-se o número de replicações para diminuir o ruído para um nível satisfatório.

Considerando que o nível de diminuição do ruído em relação à quantidade de replicações é um processo exponencial, para diminuir significativamente o ruído é necessário uma grande quanti- 
dade de replicações. Sabe-se da teoria da probabilidade que o desvio padrão é inversamente proporcional à raiz do número de amostras. Se o desvio observado do sinal é $\sigma$, seria reduzido para $\frac{\sigma}{\sqrt{N}}$ em $N$ amostras. Isto permite prever a quantidade de replicações a serem executadas. Desse modo, foram realizadas 300 replicações, que para este experimento, demonstrou ser suficiente para expor a média do comportamento do sistema. No gráfico 4.4 é possível verificar nitidamente a diminuição do ruído, além do realçamento do degrau causado na entrada. A saída também sofreu diminuição no nível de ruído, demonstrando claramente o efeito causado pela mudança brusca na carga de trabalho, ilustrada no gráfico 4.5 .

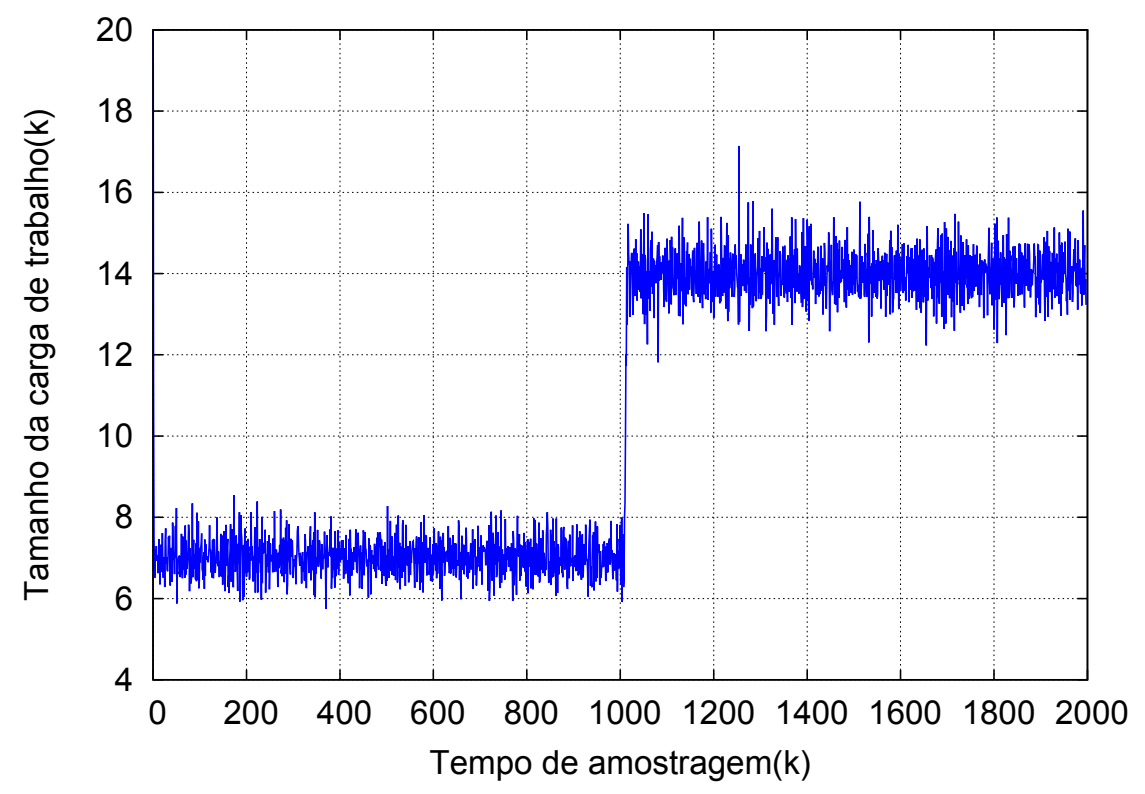

Figura 4.4: Carga de trabalho (W) imposta ao sistema.

\subsection{Modelo ARX}

Utilizando a técnica descrita por Parekh et al. (2002) para identificação de sistemas, e tendo como objetivo modelar o comportamento do sistema por uma equação de diferenças de segunda ordem, é utilizado o modelo ARX para descrever a dinâmica do sistema. O ARX é um modelo autorregressivo (AR) com uma entrada exógena, seu modelo de segunda ordem é mostrado na equação 4.2. Os parâmetros $a_{1}$ e $a_{2}$ indicam a influência das saídas anteriores e $b_{1}$ e $b_{2}$ a influência das entradas.

$$
\begin{gathered}
\hat{y}(k+1)=a_{1} \hat{y}(k)+a_{2} \hat{y}(k-1)+b_{1} u(k)+b_{2} u(k-1) \\
\text { autogressivo }=a_{1} \hat{y}(k)+a_{2} \hat{y}(k-1) \\
\text { entradaX }=b_{1} u(k)+b_{2} u(k-1)
\end{gathered}
$$




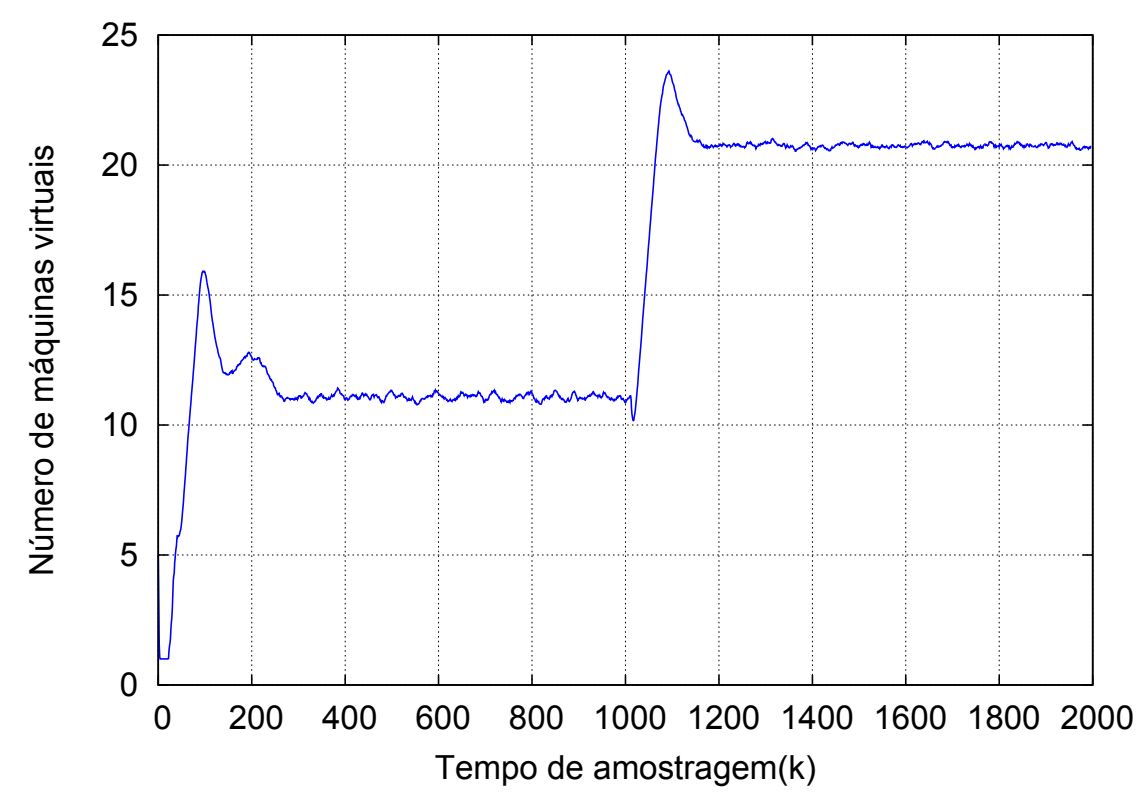

Figura 4.5: Número de máquinas virtuais previstas pelo controlador.

Para a estimação dos parâmetros do modelo, é utilizado o método dos mínimos quadrados polinomial. É importante que o valor inicial dos sinais de entrada e saída do sistema se iniciem em zero, conforme explicado na próxima seção. Após a estimação dos parâmetros, tem-se o modelo ARX parametrizado demonstrado na equação 4.3.

$$
\hat{y}(k+1)=1.546 y(k)-0.5559 y(k-1)+0.005821 u(k)+0.008061 u(k-1)
$$

\subsection{Função de transferência}

A manipulação de sinais, como a adição, subtração e o deslocamento do sinal no tempo pode envolver operações complexas no domínio do tempo. Para sinais discretos, um método conhecido de representação mais conveniente é o da transformada $\mathrm{Z}$, que atua no domínio da frequência, sendo o equivalente discreto da transformada de Laplace (Hellerstein et al., 2004). Nesse domínio, um sinal é representado por uma soma de seus valores, sendo utilizado a variável $z$ para indicar o atraso no tempo. Conforme equação 4.4. Seu equivalente no domínio da transformada $\mathrm{Z}$ é representado pela equação 4.5 .

$$
\begin{gathered}
u(k)=u(0), u(1), u(2), u(3), u(4), \ldots u(n) \\
U(z)=u(0) z^{0}+u(1) z^{-1}+u(2) z^{-2}+\ldots=\sum_{k=0}^{\infty} u(k) z^{-k}
\end{gathered}
$$


Essa representação permite fazer manipulações de forma simples. O deslocamento do sinal no tempo é feito pela multiplicação por $z^{-1}$ para atrasar o sinal. A combinação de sistemas é feita pela multiplicação de suas equações. A conversão de equações no domínio do tempo é facilitada por algumas propriedades da transformada $Z$. Primeiramente, a transformada $Z$ é uma função linear, dessa forma, se no domínio do tempo um sinal é multiplicado por uma constante, no domínio $\mathrm{Z}$ esse sinal é multiplicado pela mesma constante. Se dois sinais são somados no domínio do tempo, do domínio Z eles também são somados. O adiantamento do sinal no domínio do tempo $u(k+1)$, no domínio $\mathrm{Z}$ é necessário considerar as condições iniciais, dessa forma ficando $z U(z)-z u(0)$ para um deslocamento de $z^{1}$. Desse modo, deslocando por $z^{n}$ considera as condições iniciais $u(0),) \ldots, u(n-1)$.

Aplicando a transformada $\mathrm{Z}$ no ARX de segunda ordem da equação 4.6 obtém-se a sua análoga no domínio Z, denominada função de transferência. Considerando as propriedades apresentadas da transformada na função ARX, chega-se a equação 4.7. Para simplificar a função de transferência, a condição inicial $y(0)$ é considerada nula, desse modo $y(0)=0)$. Isolando $Y(z)$ e $U(z)$ temos a equação 4.8. Dividindo $Y(z)$ por $U(z)$ obtemos a função de transferência da equação 4.9. Por fim, multiplicamos a equação por $z^{-1}$, chegando a função de transferência, representando o sistema $F$ utilizado no experimento, demonstrado na equação 4.10 .

$$
\begin{gathered}
\hat{y}(k+1)=a_{1} \hat{y}(k)+a_{2} \hat{y}(k-1)+b_{1} u(k)+b_{2} u(k-1) \\
z Y(z)-z y(0)=a_{1} Y(z)+a_{2} z^{-1} Y(z)+b_{1} U(z)+b_{2} z^{-1} U(z) \\
\left(z-a_{1}-a_{2} z^{-1}\right) Y(z)=\left(b_{1}+b_{2} z^{-1}\right) U(z) \\
\frac{Y(z)}{U(z)}=\frac{b_{1}+b_{2} z^{-1}}{z-a_{1}-a_{2} z^{-1}} \\
F(z)=\frac{b_{1} z^{-1}+b_{2} z^{-2}}{1-a_{1} z^{-1}-a_{2} z^{-2}}
\end{gathered}
$$

Devido a simplificação da função de transferência ao considerar que $y(0)=0$, é importante que para a estimação dos parâmetros do modelo ARX, os sinais de entrada e saída sejam deslocados de modo que seu valor inicial seja igual a zero.Substituindo os parâmetros $a_{1}, a_{2}, b_{1}$ e $b_{2}$ pelos valores calculados pelo método dos mínimos quadrados, temos a função de transferência parametrizada na equação 4.11 .

$$
F(z)=\frac{0.005821 z^{-1}+0.008061 z^{-2}}{1+-1.546 z^{-1}+0.5559 z^{-2}}
$$




\subsection{Avaliação do modelo}

Utilizando a função de transferência do sistema, equação 4.11, e aplicando como entrada para averiguar o seu ajuste, a média da carga de trabalho $(W)$ apresentada no gráfico 4.4 , foi obtido a saída da função de transferência apresentada no gráfico 4.6, comparando-a com a média da saída real do sistema utilizada para encontrar os parâmetros da função. No gráfico, apesar da função ter se ajustado aos períodos estacionários da saída, no instante onde ocorreu o degrau, ele não se ajustou ao pico causado pelo mesmo. Isso demonstra a insuficiência de um modelo de segunda ordem para acomodar o ruído estocástico apresentado na média da carga de trabalho, apesar da utilização de 300 réplicas. Devido ao aumento exponencial no número de replicações necessários para suavizar a entrada e saída, é vantajoso a utilização de um filtro para esse fim.

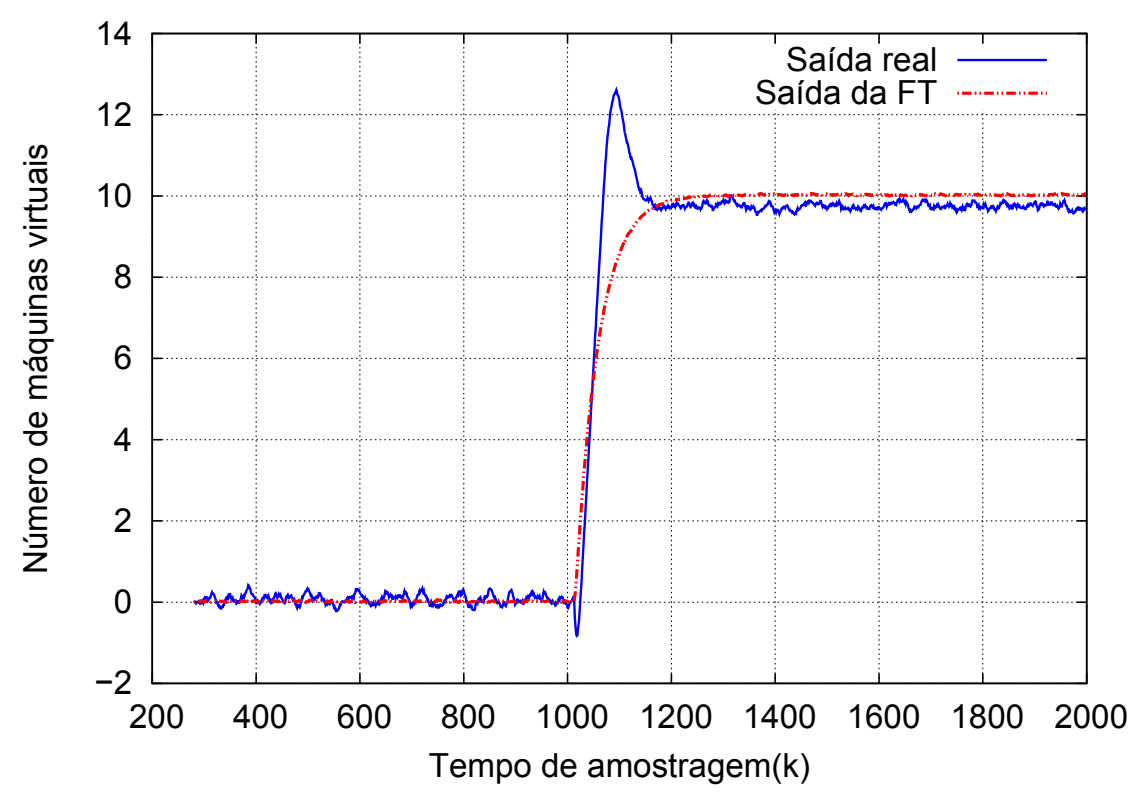

Figura 4.6: Saída prevista pela função de transferência e saída real do sistema

\subsection{Tratamento de sinais}

Para que o modelo se ajuste corretamente à saída do controlador, foi necessária a utilização de um filtro para remover a estocasticidade da variável de entrada e, por consequência, também da saída.

Assim, considerou-se um processo estocástico estacionário e o sinal de interesse são os valores médios no tempo. Foi utilizado um filtro FIR (Finite Impulse Response) passa-baixa, que é bastante utilizado na área de processamento de sinais digitais para redução de ruído. O filtro FIR é descrito por um conjunto de $p$ coeficientes polinomiais $h_{0}, \ldots h_{p-1}$, e sua saída é dada exclusivamente pelos últimos $p$ termos da entrada como mostrado na equação 4.12 . 


$$
y(n)=\sum_{i=0}^{p-1} h_{i} x(n-1)
$$

O filtro foi projetado utilizando uma janela de Hamming (Tan e Jiang, 2013), com $p=40$ e frequência de corte de $100 \mathrm{~Hz}$ (10\% da frequência máxima), obtidos empiricamente. Estes parâmetros apresentaram bons resultados, eliminando consideravelmente o ruído nos sinais de entrada e saída. Pode-se observar a diminuição do ruído na entrada $W$ pela comparação dos espectros de frequências na figura 4.7 .

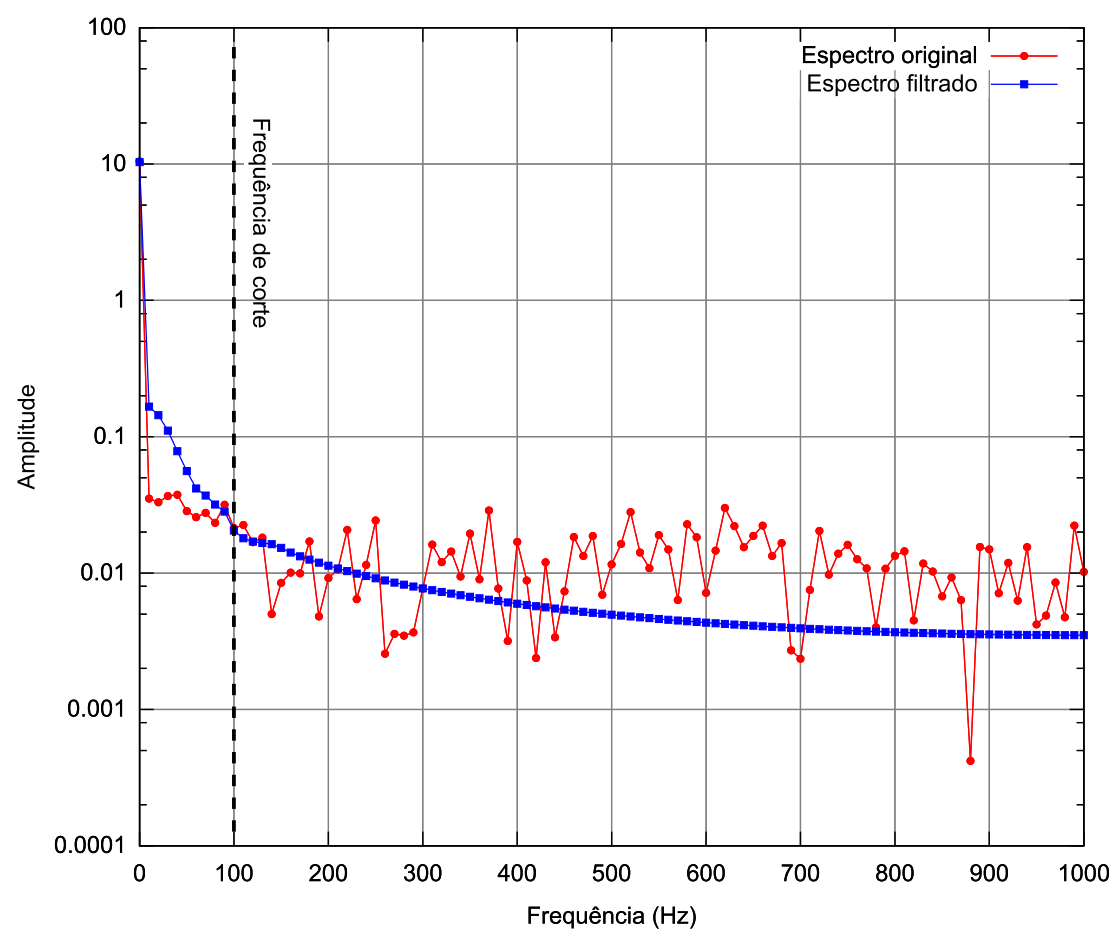

Figura 4.7: Ruído em W após utilizar o filtro em relação a W sem filtro.

\subsection{Avaliação do modelo com filtro}

Utilizando as variáveis filtradas para calcular os parâmetros do modelo ARX de segunda ordem na equação 4.6, foram obtidos novos valores aplicados a função de transferência. A função de transferência resultante dos valores filtrados é apresentado na equação 4.13.

$$
F(z)=\frac{-0.01024 z^{-1}+0.01312 z^{-2}}{1-1.96 z^{-1}+0.9617 z^{-2}}
$$

O gráfico 4.8 apresenta a saída resultante da função de transferência quando estimulado pela média filtrada da carga de trabalho. Seu resultado é confrontado com a saída real do sistema. Por inspeção visual, é possível notar uma melhoria qualitativa no ajuste. A utilização do filtro permitiu 
a função se ajustar ao degrau ocorrido, além de causar uma melhora no ajuste do período estacionário após o degrau. Contrapondo a saída real do sistema com a prevista pela função de transferência, demonstrado no gráfico 4.9, observa-se o ajuste nos períodos estacionários e transiente. No gráfico, se observa o momento de transição do período estacionário inicial para o estacionário após o degrau. Nota-se que não houve sobreposição completa no período transiente, como o gráfico 4.8 demonstra.

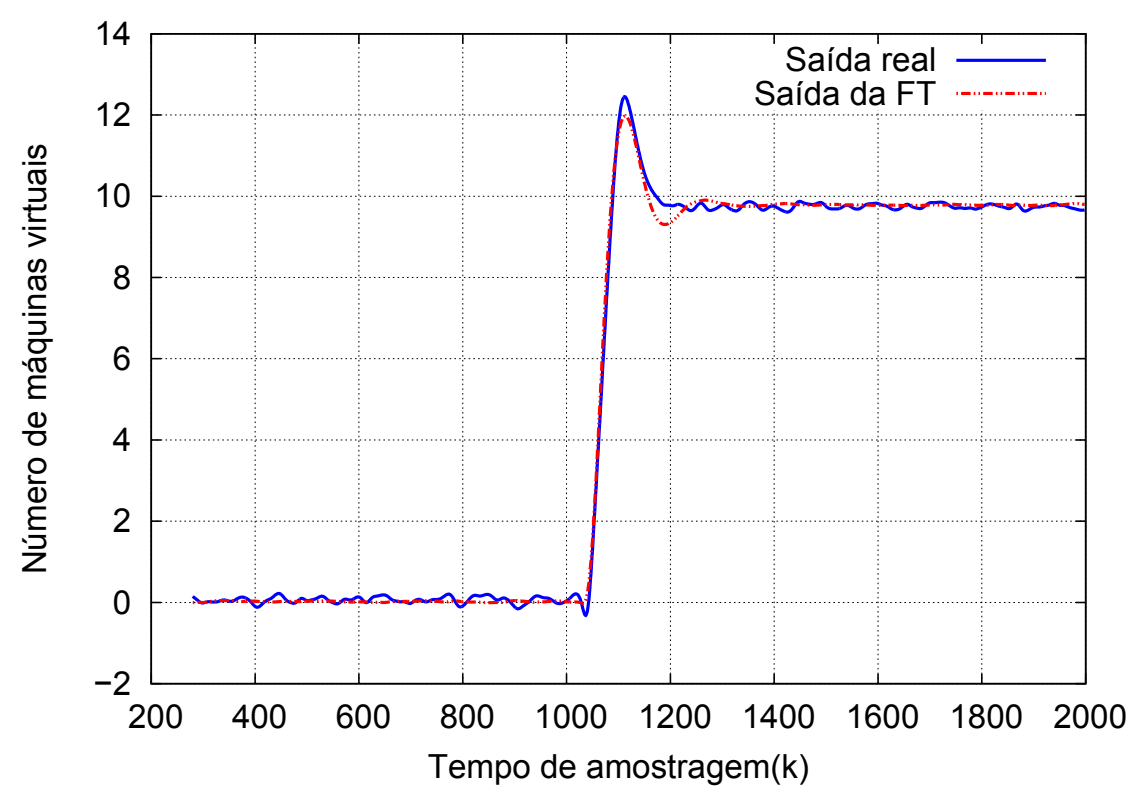

Figura 4.8: Saída prevista pela função de transferência e saída real.

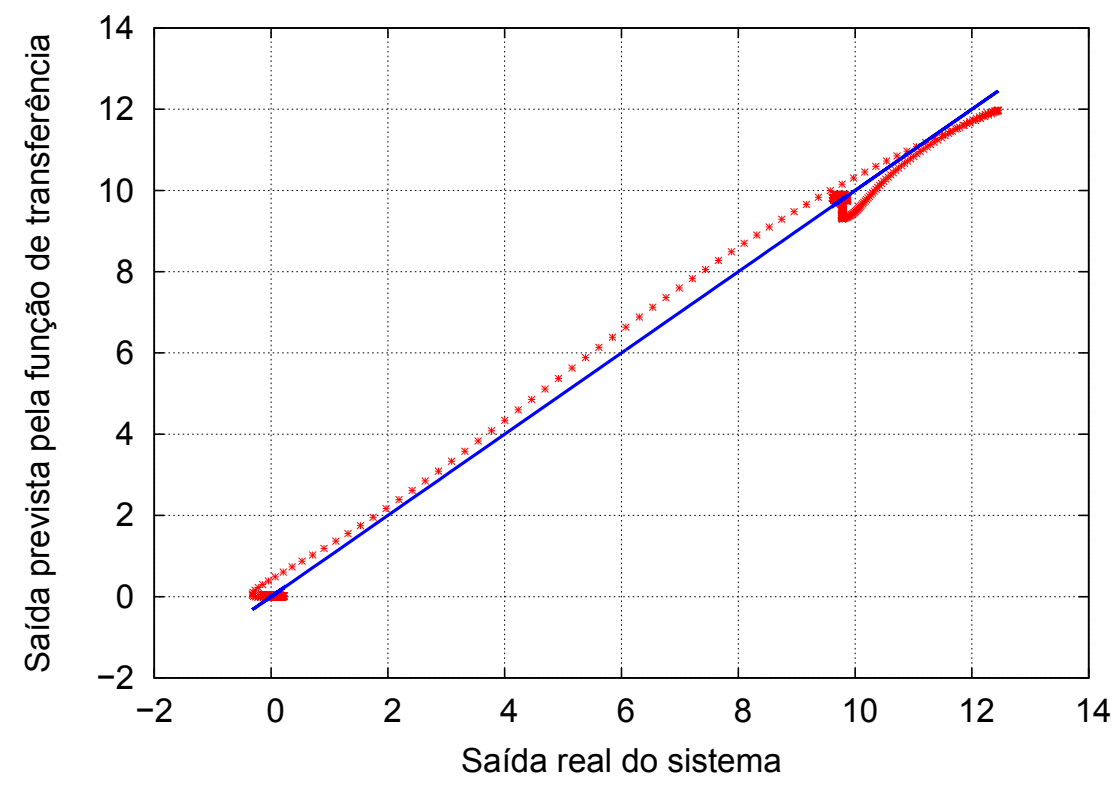

Figura 4.9: Saída prevista pela função de transferência contraposta à saída real. 
A adequação do modelo também é verificado numericamente na tabela 4.1. O baixo valor do RMSE indica o bom ajuste do modelo. O valor da variabilidade $R^{2}$ próximo de um, e a forte correlação positiva da entrada em relação a saída prevista também indicam o bom ajuste do modelo.

Tabela 4.1: Valores de $R^{2}, R M S E$ e correlação entre entrada e a saída do modelo estimado.

\begin{tabular}{lc}
\hline Teste & Valor calculado \\
\hline \hline$R^{2}$ & 0.99900 \\
$R M S E$ & 0.15429 \\
Correlação & 0.97332 \\
\hline
\end{tabular}

\subsection{Caracterização de sobrepassagem e tempo de as- sentamento}

Duas propriedades fundamentais de sistemas de segunda ordem são a razão de amortecimento $\zeta$ e a frequência natural $\omega$. Em um sistema massa-mola, $\zeta$ corresponde ao coeficiente de amortecimento da mola, que define a quantidade de energia que a mola perde por metro; e $\omega$ corresponde à frequência natural da mola, que ela exibiria na ausência de outras forças (Ogata, 2009).

Tais características são definidas para sistemas de tempo contínuo, que são analisados pela transformada de Laplace. Dada a função de transferência $G(s)$ de um sistema de segunda ordem contínuo, podemos realizar algumas caracterizações teóricas observando seus polos, isto é, as raízes do denominador. Na forma canônica de uma função de transferência contínua de $2^{\mathrm{a}}$ ordem, observamos

$$
\begin{aligned}
G(s)=\frac{N(s)}{D(s)} & =\frac{N(s)}{s^{2}+a s+b} \\
& =\frac{N(s)}{s^{2}+2 \zeta \omega s+\omega^{2}}
\end{aligned}
$$

isto é, $\omega=\sqrt{b}$ e $\zeta=a /(2 \sqrt{b})$.

Para obter estes parâmetros para um sistema discreto, definido no espaço da transformada Z, é necessário realizar uma aproximação para seu comportamento em tempo contínuo. A transformação bilinear, dada na equação 4.14 , fornece uma aproximação de $1^{\text {a }}$ ordem:

$$
\begin{aligned}
z & =\exp (s T) \\
& =\frac{\exp (s T / 2)}{\exp (-s T / 2)} \\
& \approx \frac{1+s T / 2}{1-s T / 2}
\end{aligned}
$$


onde $T$ é o período de amostragem do sinal discreto.

Quando a razão de amortecimento $\zeta$ é inferior a um valor crítico, o sistema se mostra sub amortecido; quando excitado por uma entrada degrau, o sinal apresenta oscilações decrescentes até atingir um estado estacionário. Podemos determinar características teóricas do sistema a partir da razão de amortecimento $\zeta$ e da frequência natural $\omega$.

Neste caso é de interesse caracterizar a sobrepassagem (overshoot) $M_{p}$, definida como o máximo valor alcançado pelo sinal, assim como a sobrepassagem relativa $O_{p}$, dada como a razão da diferença do valor de pico em relação ao valor estacionário do sinal $x_{\infty}$, dado na equação 4.15. Esta é uma medida de extrema relevância para sistemas de segunda ordem, pois impacta no dimensionamento de recursos e no máximo sinal suportado.

$$
O_{p}=\frac{M_{p}-x_{\infty}}{x_{\infty}}=\exp \left(-\frac{\pi \zeta}{\sqrt{1-\zeta^{2}}}\right)
$$

Também, é interessante caracterizar o tempo de assentamento (settling time) $T_{s}$, definido como o máximo tempo entre a perturbação e o sinal se apresentar dentro de uma certa faixa ao redor do valor estacionário. Para uma dada faixa de tolerância $\delta$, podemos obter uma aproximação teórica dada pela equação 4.16 , válida para quando $\zeta \ll 1$ :

$$
T_{S}=\frac{\ln \delta}{\zeta \omega}
$$

Tanto a sobrepassagem quanto o tempo de assentamento estão ilustrados na figura 4.10.

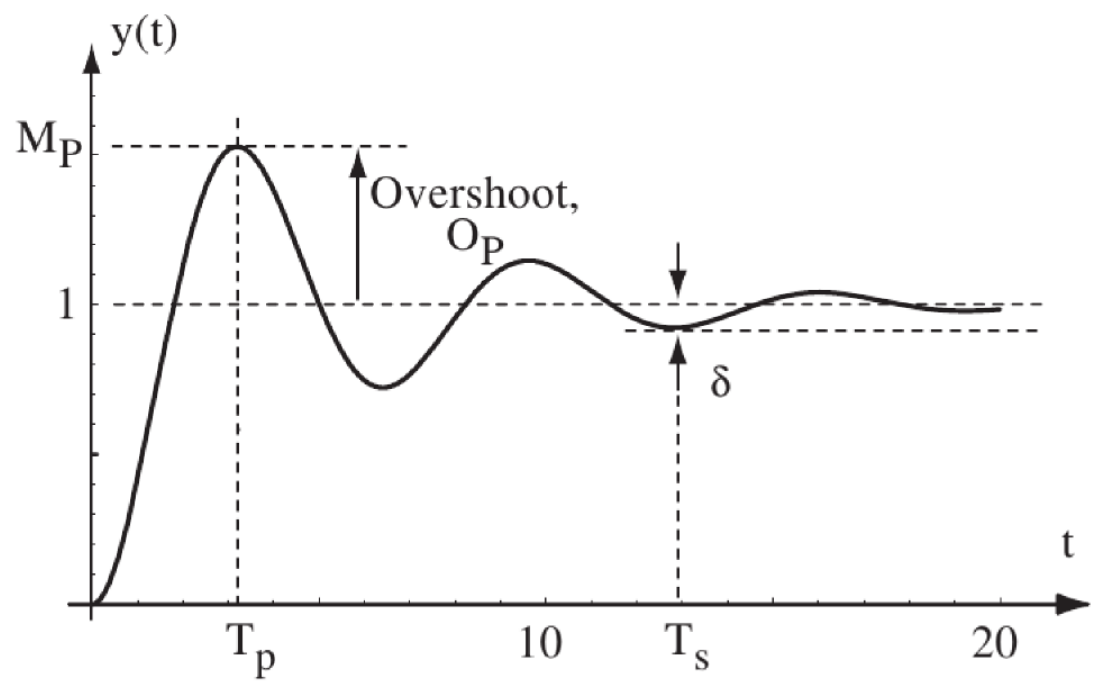

Figura 4.10: Exemplo de resposta de um sistema de $2^{\text {a }}$ ordem a uma entrada degrau.

Utilizando a transformação bilinear dada na equação 4.14, obtivemos o sistema aproximado de tempo contínuo $\tilde{G}(s)$ equivalente a $G(z)$, dado na equação 4.17 :

$$
\tilde{G}(s)=\frac{0.005957 s^{2}-0.001338 s+2.938 \times 10^{-5}}{s^{2}+0.003906 s+1.734 \times 10^{-5}}
$$


Com isto pudemos extrair as características teóricas do sistema, listados na coluna "Teórico"da Tabela 4.10. Mensuramos a sobrepassagem e tempo de assentamento do sinal de saída real e comparamos com a saída do modelo analítico obtido, como colocados na tabela 4.10. Notamos um bom ajuste da previsão da sobrepassagem e um bom ajuste da previsão do tempo de assentamento. Esses valores podem ser melhorados de acordo com a necessidade ajustando o modelo obtido. Também é apresentado valores teóricos resultantes de um degrau unitário no modelo.

Tabela 4.2: Comparação de características do modelo analítico com o mensurado do sistema.

\begin{tabular}{cc|cccc}
\hline & $\begin{array}{c}\text { Teórico } \\
\text { degrau unitário }\end{array}$ & $\begin{array}{c}\text { Modelo } \\
\text { analítico }\end{array}$ & $\begin{array}{c}\text { Mensurado } \\
\text { do sistema }\end{array}$ & $\begin{array}{c}\text { Erro } \\
\text { absoluto }\end{array}$ & $\begin{array}{c}\text { Erro } \\
\text { relativo }\end{array}$ \\
\hline \hline$(\omega)$ & $4.164 \times 10^{-3} \mathrm{~Hz}$ & - & - & - & - \\
$(\zeta)$ & 0.469 & - & - & - & - \\
$\left(O_{p}\right)$ & $18.86 \%$ & $22.36 \%$ & $27.76 \%$ & $5.4 \%$ & $24.15 \%$ \\
$\left(T_{s}\right)$ & $2003 \mathrm{rtu}$ & $1620 \mathrm{rtu}$ & $1310 \mathrm{rtu}$ & $310 \mathrm{rtu}$ & $23.66 \%$
\end{tabular}




\section{CAPítulo \\ 5}

Conclusões

Este trabalho apresenta os resultados de um estudo sobre previsão de sobrecarga transiente em sistemas computacionais. A principal contribuição da pesquisa é a formulação de um método para modelagem do sistema na forma de uma representação analítica capaz de reproduzir o comportamento dinâmico do sistema. Foi utilizado como estudo de caso um sistema investigado em um trabalho anterior (Nobile, 2013), o qual simula uma infraestrutura de computação em nuvem. Um dos resultados desse trabalho é a viabilidade de representar a complexidade de um datacenter por um modelo simples, especificamente um sistema dinâmico linear e invariante no tempo, possível de ser tratado por técnicas clássicas de análise de sistemas lineares.

O referido estudo anterior implementa um mecanismo adaptativo de alocação de recursos elásticos em que o número de máquinas virtuais é gerenciado por um controlador regulatório cuja meta é manter constante a taxa de utilização média do conjunto. Foi demonstrado (Nobile, 2013) que o sistema controlado possui uma dinâmica de primeira ordem; o laço de realimentação responsável pela adaptação (feedback) em malha fechada eleva a ordem do sistema. Esse conhecimento a priori da natureza dinâmica do sistema, motivou a formulação da hipótese de pesquisa do trabalho, a de considerar uma aproximação de segunda ordem. Procedimentos experimentais foram conduzidos para a verificação empírica dessa suposição, com resultado positivo. O sistema de referência foi, então, tomado como uma "caixa preta" e submetida a ensaios empíricos de comparação entre entrada e saída para derivar um modelo analítico, construído segundo um processo indutivo, e não dedutivamente a partir dos princípios elementares.

O método de identificação do sistema pode ser sintetizado nos seguintes passos.

a) preparação do sistema a ser estudado, seja um protótipo físico ou um modelo simulado; 
b) obtenção de uma carga de trabalho estacionária, seja sintética ou obtida de logs;

c) geração de uma perturbação em forma de degrau na carga de trabalho;

d) reamostragem da carga de entrada e extração da média entre as séries a fim de reduzir a intensidade do ruído;

e) aplicação de um filtro passa-baixas para suavização dos ruídos estocásticos e obtenção do sinal estacionário dos valores médios da carga;

f aplicação de um offset relativo ao sinal para transladar a entrada inicial ao nível zero;

g) aplicação do sinal filtrado à entrada do sistema-referência e mensuração do sinal de saída;

h) verificação qualitativa, por inspeção, das hipóteses de dinâmica de primeira ou segunda ordem;

i) construção de um modelo autorregressivo com variável exógena (ARX) de ordem equivalente;

j) parametrização do modelo ARX a partir dos dados experimentais utilizando um método de ajuste como, por exemplo, o método dos mínimos quadrados;

k) aplicação da transformada $\mathrm{Z}$ sobre o modelo ARX para formulação da função de transferência;

1) aplicar à função de transferência o sinal experimental (filtrado e transladado) e obter a saída predita;

m) comparar a saída do sistema-referência com a saída prevista utilizando testes estatísticos;

n) se o ajuste foi bom, o modelo é considerado, do contrário, as hipóteses devem ser revistas e reavaliadas.

Com a função de transferência é possível obter, quer por métodos numéricos (p.ex. utilizando uma ferramenta como Octave ou Matlab ${ }^{1}$ ), quer pela análise de polos e zeros da função, prever o overshoot e tempo de assentamento, que são dois parâmetros importantes para descrever a sobrecarga transiente.

No desenvolvimento do trabalho foram feitas algumas escolhas práticas sobre a carga de trabalho. Para realização dos experimentos, a carga de trabalho foi parametrizada por uma relação constante entre tempo médio de processamento e tempo médio entre chegadas, à qual é superposta a uma perturbação na forma de um degrau. Essa configuração resulta em uma demanda do tipo Poisson, onde os tempos de chegada e de processamento são dados por distribuições exponenciais.

A obtenção dos parâmetros do modelo dinâmico e validação dos resultados mediante o confronto com o sistema-referência foram feitos sob essa condição. Todavia, uma vez que a abordagem assume um processo estocástico estacionário, no qual o valor médio da entrada e saída

\footnotetext{
${ }^{1}$ www.matlab.com
} 
são tomados como sinais (e para isso foi realizado o tratamento de sinais por filtragem de altas frequências), o aspecto estocástico não é relevante, de modo que as conclusões gerais são válidas independentemente da distribuição que represente a carga. Também, acerca da perturbação em forma de degrau, uma vez obtida a função de transferência, e dentro da faixa de operação em que é válida a aproximação do comportamento linear e invariante no tempo, o modelo pode ser utilizado para prever a sobrecarga transiente devido a outras formas de perturbações, sejam degrau, oscilações sazonais ou rajadas. Numericamente isso pode ser feito aplicando-se a entrada (filtrada e transladada para estado inicial zero) à função de transferência. Algebricamente, há técnicas de análise de sinais que podem ser empregadas para obter a representação analítica da entrada, a qual pode ser multiplicada pela função de transferência para obter a resposta no domínio Z, e depois analisadas diretamente ou transformadas para o domínio do tempo.

Conclui-se, portanto, que, válidas as hipóteses sobre o sistema, o modelo analítico permite prever sobrecargas decorrentes de variações bruscas de carga de trabalho, ainda aquelas de duração transitória, e que se dissipam, mas que podem implicar em um esforço superior àquele exigido do sistema nas condições estacionárias inicias e finais. Em sistemas com recurso limitados, esses picos e seus tempos de duração podem ter um impacto considerável no desempenho geral do sistema. O presente trabalho avança na aplicação de técnicas apropriadas para a obtenção de modelos capazes de descrever esse comportamento e serem utilizados para predições. Avança também na exploração do emprego de modelos dinâmicos em sistemas computacionais, campo com vasto terreno para contribuições, e que começa a tornar-se relevante em função da proliferação de sistemas distribuídos de larga escala. Os resultados corroboram a motivação de que a avaliação de capacidade estática de sistemas computacionais, restritas ao regime estacionário, podem ser complementadas pela avaliação de capacidade dinâmica, que englobam o regime transiente decorrente da dinâmica de sistemas desse tipo.

Como trabalhos futuros, são elencados alguns desdobramentos e questões a serem investigadas. Existem técnicas de análise em domínio de frequência que podem ser utilizadas para quantificar critérios de escolha de tempos de amostragem e adequação da hipótese de linearidade. Essas podem ser incorporadas ao método como passo inicial de pré-avaliação e calibração de alguns valores que, neste trabalho, foram ajustados empiricamente.

O presente estudo foi realizado em sequência a trabalhos anteriores alinhados ao mesmo tema de pesquisa, e em paralelo a outras iniciativas que investigam identificação de capacidade dinâmica em sistemas computacionais. Mamani (2012) desenvolve um protótipo físico que implementa a arquitetura de Nobile (2013), utilizada como estudo de caso neste trabalho, a fim de avaliar a consistência dos resultados simulados com os aferidos em um sistema real. Pereira (2013) pesquisa uma metodologia para projeto de políticas de controle de admissão capazes de evitar sobrecargas transientes. Os resultados do presente trabalho contribuem para ambos os projetos com a formulação e avaliação da metodologia para modelagem do sistema e do seu comportamento dinâmico. 



\section{Referências}

Abdelzaher, T.; DiaO, Y.; Hellerstein, J. L.; Lu, C.; Zhu, X. Introduction to Control Theory And Its Application to Computing Systems. In: LiU, Zhen And XiA, C. H., ed. Performance Modeling and Engineering, cáp. 7, Springer US, p. 185-215, 2008.

Abdelzaher, T.; Stankovic, J.; Lu, C.; Zhang, R.; Lu, Y. Feedback performance control in software services. 2003.

Disponível em: citeseer.ist.psu.edu/abdelzaher03feedback.html

AGUIRRE, L. Introdução à identificação de sistemas - técnicas lineares e não-lineares aplicadas a sistemas reais. 3rd ed. Editora UFMG, 2007.

AmAzon Amazon ec2 faqs. http://aws.amazon.com/ec2/faqs, accessado: 31/07/2014, 2014.

Buyya, R.; RANJAN, R.; CALheiros, R. N. Modeling and simulation of scalable cloud computing environments and the cloudsim toolkit: Challenges and opportunities. In: 2009 International Conference on High Performance Computing \& Simulation, IEEE, 2009.

Egami, K.; Matsumoto, S.; NAKamura, M. Ubiquitous cloud: Managing service resources for adaptive ubiquitous computing. In: 2011 IEEE International Conference on Pervasive Computing and Communications Workshops (PERCOM Workshops), IEEE, 2011, p. 123-128. Disponível em: http://ieeexplore.ieee.org/lpdocs/epic03/wrapper. htm? arnumber $=5766853$

HARJUNKOSKI, I.; NYSTRÖM, R.; HORCH, A. Integration of scheduling and control-theory or practice? Computers \& Chemical Engineering, v. 33, n. 12, p. 1909-1918, 2009.

HAYES, B. Cloud computing. Communications of the ACM, v. 51, n. 7, p. 9, 2008.

Disponível em: http://portal.acm.org/citation.cfm?doid=1364782. 1364786 
Hellerstein, J.; Parekh, S.; Griffith, R.; Kaiser, G.; Phung, D. A control theory foundation for self-managing computing systems. IEEE Journal on Selected Areas in Communications, v. 23, n. 12, p. 2213-2222, 2005.

Disponível em: http://ieeexplore.ieee.org/lpdocs/epic03/wrapper. htm? arnumber $=1546093$

Hellerstein, J. L.; Diao, Y.; PARekh, S.; Tilbury, D. M. Feedback Control of Computing Systems. 1 ed. Hoboken, NJ, USA: John Wiley \& Sons, Inc., 429 p., 2004.

Disponível em: http://doi.wiley.com/10.1002/047166880X

Hellerstein, J. L.; Kohlhoff, K. J.; Konerding, D. E. Science in the Cloud: Accelerating Discovery in the 21st Century. IEEE Internet Computing, v. 16, n. 4, p. 64-68, 2012.

Disponível em: http://ieeexplore.ieee.org/xpl/articleDetails.jsp? arnumber $=6257372$

Henriksson, D.; Lu, Y.; ABDElZAher, T. Improved prediction for web server delay control. Proceedings. 16th Euromicro Conference on Real-Time Systems, 2004. ECRTS 2004., p. 61-68, 2004.

Disponível em: http://ieeexplore.ieee.org/lpdocs/epic03/wrapper. htm? arnumber $=1311001$

Horn, P. Autonomic computing: IBM's Perspective on the State of Information Technology. 2001.

Disponível em: http://www.research.ibm.com/autonomic/manifesto/ autonomic_computing.pdf

JAIN, R. K. The Art of Computer Systems Performance Analysis: Techniques for Experimental Design, Measurement, Simulation, and Modeling. Wiley Computer Publishing, John Wiley \& Sons, Inc., 685 p., 1991.

Disponível em: http://books.google.com.br/books/about/The_art_of_ computer_systems_performance.html?id=t91QAAAAMAAJ\&redir_esc

KAmra, A.; Misra, V.; NAhUM, E. Yaksha: a self-tuning controller for managing the performance of 3-tiered web sites. Twelfth IEEE International Workshop on Quality of Service, 2004. IWQOS 2004., p. 47-56, 2004.

Disponível em: http://ieeexplore.ieee.org/lpdocs/epic03/wrapper. htm? arnumber $=1309356$

Kannan, S.; Gavrilovska, A.; Schwan, K. Cloud4Home - Enhancing Data Services with @ Home Clouds. In: 201131 st International Conference on Distributed Computing Systems, IEEE, 2011, p. 539-548.

Disponível em: http://ieeexplore.ieee.org/lpdocs/epic03/wrapper. htm? arnumber $=5961767$ 
Lim, H.; BABU, S.; Chase, J.; PAREKh, S. Automated control in cloud computing: challenges and opportunities. In: Proceedings of the 1st workshop on Automated control for datacenters and clouds, ACM, 2009, p. 13-18.

Lu, C.; Abdelzaher, T. F.; Stankovic, J. A.; Son, S. H. A feedback control approach for guaranteeing relative delays in web servers. In: RTAS '01: Proceedings of the Seventh Real-Time Technology and Applications Symposium (RTAS '01), Washington, DC, USA: IEEE Computer Society, 2001, p. 51.

Lu, C.; Lu, Y.; Abdelzaher, T. F.; Stankovic, J. A.; Son, S. H. Feedback Control Architecture and Design Methodology for Service Delay Guarantees in Web Servers. IEEE Transactions on Parallel and Distributed Systems, v. 17, n. 9, p. 1014-1027, 2006.

Disponível em: http://ieeexplore.ieee.org/lpdocs/epic03/wrapper. htm?arnumber $=1668065$

Lu, C.; Stankovic, J. A.; Son, S. H.; TaO, G. Feedback Control Real-Time Scheduling : Framework, Modeling, and Algorithms. Real-Time Systems, v. 23, p. 85-126, 2002.

Lu, C.; Stankovic, J. A.; TaO, G.; Son, S. H. Design and evaluation of a feedback control edf scheduling algorithm. In: RTSS '99: Proceedings of the 20th IEEE Real-Time Systems Symposium, Washington, DC, USA: IEEE Computer Society, 1999, p. 56.

Lu, Y.; Abdelzaher, T.; Lu, C.; Sha, L.; LiU, X. Feedback control with queueing-theoretic prediction for relative delay guarantees in web servers. In: Real-Time and Embedded Technology and Applications Symposium, 2003. Proceedings. The 9th IEEE, 2003, p. 208-217.

LunZe, J.; Lehmann, D. A state-feedback approach to event-based control. Automatica, v. 46, n. 1, p. 211-215, 2010.

MAMANi, E. L. C. Uma abordagem de controle realimentado para gerenciamento autonômico de datacenters baseada em algoritmos auto-adaptativos distribuídos de balanceamento de carga em computação em nuvem. Tese de Doutoramento, ICMC USP, monografia de qualificação, 2012.

MilleR, B. The autonomic computing edge: Can you chop up autonomic computing? 2008.

Disponível em: http://www.ibm.com/developerworks/library/ac-edge4/ index.html

Müller, H.; Kienle, H.; Stege, U. Autonomic Computing Now You See It, Now You Don't: Design and Evolution of Autonomic Software Systems. In: De-Lucia, A.; Ferrucci, F., eds. Software Engineering, v. 5413/2009, Salerno, Italy: Springer-Verlag Berlin Heidelberg, p. 32-54, 2009.

Disponível em: http://dx.doi.org/10.1007/978-3-540-95888-8_2 
Nobile, P. N. Projeto de um broker de gerenciamento adaptativo de recursos em computação em nuvem baseado em técnicas de controle realimentado. Tese de Doutoramento, ICMC-USP, 2013.

OCTAVE COMMUNITY GNU Octave 3.8.1. 2014.

Disponível em: www.gnu.org/software/octave/

Ogata, K. Modern Control Engineering. 5th ed. Prentice-Hall, 912 p., 2009.

OGATA, K. Modern control engineering. Instrumentation and controls series, 5 th ed. Prentice Hall, 905 p., 2010.

Disponível em: http: / /books.google.com.br/books? id=Wu5GpNAel zkC

Parekh, S.; GAndhi, N.; Hellerstein, J. Using control theory to achieve service level objectives in performance management. Real-Time Systems, v. 23, p. 127-141, 2002.

Disponível em: http://www.springerlink.com/index/NKQN08V9P482PKXH. $\operatorname{pdf}$

PEREIRA, L. A. J. Uma metodologia de planejamento de capacidade para política de admissão de controle em sistemas computacionais sob carga variável baseada em avaliação de desempenho em regime transiente. Tese de Doutoramento, ICMC USP, monografia de qualificação, 2013.

QIN, W.; WAng, Q. Modeling and Control Design for Performance Management of Web Servers Via an LPV Approach. IEEE Transactions on Control Systems Technology, v. 15, n. 2, p. 259-275, 2007.

Disponível em: http://ieeexplore.ieee.org/lpdocs/epic03/wrapper. htm? arnumber $=4105947$

Sha, L.; Abdelzaher, T.; Arzén, K.-E.; Cervin, A.; Baker, T.; Burns, A.; Buttazzo, G.; CACCAmo, M.; LehoczKy, J.; MoK, A. K. Real time scheduling theory: A historical perspective. Real-Time Syst., v. 28, n. 2-3, p. 101-155, 2004.

TAN, L.; JiAng, J. Digital signal processing: fundamentals and applications. Academic Press, 2013.

Wang, Y.; Wang, X.; Chen, M.; Zhu, X. Partic: Power-aware response time control for virtualized web servers. Parallel and Distributed Systems, IEEE Transactions on, v. 22, n. 2, p. 323-336, 2011.

XIA, F.; SUn, Y.; TIAN, Y. Feedback scheduling of priority-driven control networks. Computer Standards \& Interfaces, v. 31, n. 3, p. 539-547, 2009. 
XIONG, P. Dynamic management of resources and workloads for RDBMS in cloud. In: Proceedings of the on SIGMOD/PODS 2012 PhD Symposium - PhD '12, New York, New York, USA: ACM Press, 2012, p. 63.

Disponível em: http://dl.acm.org/citation. cfm?doid=2213598.2213614

Zhu, X.; Uysal, M.; Wang, Z.; Singhal, S.; Merchant, A.; Padala, P.; Shin, K. What does control theory bring to systems research? ACM SIGOPS Operating Systems Review, v. 43, n. 1, p. 62-69, 2009. 